COMMUNICATIONS IN

ANALYSIS AND GEOMETRY

Volume 15, Number 1, 29-76, 2007

\title{
A Monge-Ampère-type equation motivated by string theory
}

\author{
Ji-Xiang Fu And Shing-Tung YaU
}

In this paper, we solve a Monge-Ampère-type equation by the continuity method. This equation is motivated by the superstring theory.

\section{Introduction}

There is a rich class of non-Kähler complex manifolds for dimension greater than two. It is therefore important to construct canonical geometry on such manifolds. Since non-Kähler geometry is not as intuitive as Riemannian geometry, it has been difficult to find a reasonable class of Hermitian metrics that exhibit rich geometry. We believe that metrics motivated by theoretic physics should have good properties. This is especially true for those metrics that are supersymmetric. The work of Strominger did provide such a candidate. In this paper, we provided a smooth solution for Strominger's system. While it is not hard to generalize such system to elliptic fibration over general Calabi-Yau manifolds based on the methods developed here, we shall restrict ourselves to complex three-dimensional examples. We shall discuss the higher dimensional examples in other paper. In the following, we motivate the point of view from string theory.

In the original proposal for compactification of superstring, Candelas et al. [1] constructed the metric product of a maximal symmetric fourdimensional spacetime $M$ with a six-dimensional Calabi-Yau vacuum $X$ as the ten-dimensional space-time; they identified the Yang-Mills connection with the $S U(3)$ connection of the Calabi-Yau metric and set the dilaton to be a constant. Adapting the second author's suggestion of using UhlenbeckYau's theorem on constructing Hermitian-Yang-Mills connections over stable bundles [9], Witten [11] and later Horava-Witten [4] proposed to use higher rank bundles for strong coupled heterotic string theory so that the gauge groups can be $S U(4)$ or $S U(5)$.

At around the same time, Strominger [7] analyzed heterotic superstring background with spacetime supersymmetry and non-zero torsion by allowing 
a scalar "warp factor" for the spacetime metric. He considered a tendimensional spacetime that is a warped product of a maximal symmetric four-dimensional spacetime $M$ and an internal space $X$; the metric on $M \times X$ takes the form

$$
g^{0}=e^{2 D(y)}\left(\begin{array}{cc}
g_{\mu \nu}(x) & 0 \\
0 & g_{i j}(y)
\end{array}\right), \quad x \in M, y \in X
$$

the connection on an auxiliary bundle is Hermitian-Yang-Mills connection over $X$ :

$$
F \wedge \omega^{2}=0, \quad F^{2,0}=F^{0,2}=0 .
$$

Here $\omega$ is the Hermitian form $\omega=\frac{\sqrt{-1}}{2} g_{i \bar{j}} d z^{i} \wedge d \bar{z}^{j}$ defined on the internal space $X$. In this system, the physical relevant quantities are

$$
\begin{aligned}
h & =-\sqrt{-1}(\bar{\partial}-\partial) \omega, \\
\phi & =-\frac{1}{2} \log \|\Omega\|+\phi_{0}
\end{aligned}
$$

and

$$
g_{i j}^{0}=e^{2 \phi_{0}}\|\Omega\|^{-1} g_{i j}
$$

for a constant $\phi_{0}$.

In order for the ansatz to provide a supersymmetric configuration, one introduces a Majorana-Weyl spinor $\epsilon$ so that

$$
\begin{gathered}
\delta \psi_{M}=\nabla M \epsilon-\frac{1}{8} h_{M N P} \gamma^{N P} \epsilon=0, \\
\delta \lambda=\gamma^{M} \partial_{M} \phi \epsilon-\frac{1}{12} h_{M N P} \gamma^{M N P} \epsilon=0, \\
\delta \chi=\gamma^{M N} F_{M N} \epsilon=0
\end{gathered}
$$

where $\psi_{M}$ is the gravitino, $\lambda$ the dilatino, $\chi$ the gluino, $\phi$ the dilaton and $h$ the Kalb-Ramond field strength obeying

$$
d h=\frac{\alpha^{\prime}}{2}(\operatorname{tr} F \wedge F-\operatorname{tr} R \wedge R)
$$

Strominger [7] showed that in order to achieve spacetime supersymmetry, the internal six manifold $X$ must be a complex manifold with a non-vanishing 
holomorphic 3 -form $\Omega$; and the anomaly cancellation demands that the Hermitian form $\omega$ obey $^{1}$

$$
\sqrt{-1} \partial \bar{\partial} \omega=\frac{\alpha^{\prime}}{4}(\operatorname{tr} R \wedge R-\operatorname{tr} F \wedge F)
$$

and supersymmetry requires ${ }^{2}$

$$
d^{*} \omega=\sqrt{-1}(\bar{\partial}-\partial) \log \|\Omega\|_{\omega}
$$

Accordingly, he proposed the system

$$
\begin{array}{r}
F_{H} \wedge \omega^{2}=0 ; \\
F_{H}^{2,0}=F_{H}^{0,2}=0 ; \\
\sqrt{-1} \partial \bar{\partial} \omega=\frac{\alpha^{\prime}}{4}\left(\operatorname{tr} R \wedge R-\operatorname{tr} F_{H} \wedge F_{H}\right) ; \\
d^{*} \omega=\sqrt{-1}(\bar{\partial}-\partial) \ln \|\Omega\|_{\omega} .
\end{array}
$$

This system gives a solution of a superstring theory with flux that allows non-trivial dilaton field and Yang-Mills field. (It turns out $D(y)=\phi$ and is the dilaton field.) Here $\omega$ is the Hermitian form and $R$ is the curvature tensor of the Hermitian metric $\omega ; H$ is the Hermitian metric and $F$ is its curvature of a vector bundle $E$; tr is the trace of the endomorphism bundle of either $E$ or $T X$.

In [5], Li and Yau gave the first irreducible non-singular solution of the supersymmetric system of Strominger for $U(4)$ and $U(5)$ principle bundle. They obtained their solutions by perturbing around the Calabi-Yau vacuum coupled with the sum of tangent bundle and trivial line bundles. In our previous paper [2], we gave, first, examples of solutions to Strominger's system on a non-Kähler manifold, which is a torus bundle over a $K 3$ surface. In this paper, we consider the case $\alpha^{\prime}<0$. This provides an interesting system of nonlinear partial differential equations.

As in our previous paper, we construct a solution on a torus bundle over the K3 surface constructed by Goldstein and Prokushkin [3]. Let $\left(S, \omega_{S}, \Omega_{S}\right)$ be a K3 surface or complex torus with Kähler form $\omega_{S}$ and a non-vanishing

\footnotetext{
${ }^{1}$ The curvature $F$ of the vector bundle $E$ in ref. [7] is real, i.e., $c_{1}(E)=\frac{F}{2 \pi}$. But, we are used to taking the curvature $F$ such that $c_{1}(E)=\frac{\sqrt{-1}}{2 \pi} F$. So this equation has modified Equation (2.18) of ref. [7] by a minus sign.

${ }^{2}$ See Equation (56) of ref. [8], which corrects Equation (2.30) of ref. [7] by a minus sign.
} 
holomorphic $(2,0)$-form $\Omega_{S}$. Let $\omega_{1}$ and $\omega_{2}$ be anti-self-dual (1,1)-forms such that $\frac{\omega_{1}}{2 \pi}$ and $\frac{\omega_{2}}{2 \pi}$ represent integral cohomology classes. Using these two forms, Goldstein and Prokushkin constructed the non-Kähler manifold $X$ such that $\pi: X \rightarrow S$ is a holomorphic $T^{2}$-fibration over $S$ with hermitian form $\omega_{0}=\pi^{*} \omega_{S}+\frac{\sqrt{-1}}{2} \theta \wedge \bar{\theta}$ and holomorphic 3-form $\Omega=\Omega_{S} \wedge \theta$ (for the definition of $\theta$, see Section 2). Our solution to Strominger's system for $\alpha^{\prime}<0$ can be constructed as follows.

Let $L_{1}$ and $L_{2}$ be holomorphic line bundle over $S$ such that their curvature forms are given by $\sqrt{-1} \omega_{1}$ and $\sqrt{-1} \omega_{2}$, respectively. Corresponding to these curvature forms, there exist Hermitian metrics $h_{1}$ and $h_{2}$ on $L_{1}$ and $L_{2}$. Let $E=L_{1} \oplus L_{2} \oplus T^{\prime} S$ and $H_{0}=\left(h_{1}, h_{2}, \omega_{S}\right)$. Then $F_{H_{0}}=\operatorname{diag}\left(\sqrt{-1} \omega_{1}, \sqrt{-1} \omega_{2}, R_{\omega_{S}}\right)$. Let $u$ be any smooth function on $S$ and

$$
\omega_{u}=\pi^{*}\left(e^{u} \omega_{S}\right)+\frac{\sqrt{-1}}{2} \theta \wedge \bar{\theta}
$$

Then $\left(V=\pi^{*} E, \pi^{*} F_{H_{0}}, X, \omega_{u}\right)$ satisfies Strominger's Equations (1.1),(1.2) and (1.4) and we only need to consider Equation (1.3). Because $\omega_{1}$ and $\omega_{2}$ are harmonic, we can write $\omega_{1}$ and $\omega_{2}$ locally as

$$
\omega_{1}=\bar{\partial} \xi=\bar{\partial}\left(\xi_{1} d z_{1}+\xi_{2} d z_{2}\right)
$$

and

$$
\omega_{2}=\bar{\partial} \zeta=\bar{\partial}\left(\zeta_{1} d z_{1}+\zeta_{2} d z_{2}\right),
$$

where $\left(z_{1}, z_{2}\right)$ are local coordinate on $S$. Let

$$
B=\left(\begin{array}{l}
\xi_{1}+\sqrt{-1} \zeta_{1} \\
\xi_{2}+\sqrt{-1} \zeta_{2}
\end{array}\right)
$$

Using matrix $B$, we can compute curvature $R_{u}$ of the metric $\omega_{u}$ and $R_{u} \wedge R_{u}$ as in our previous paper [2]. Let $g=\left(g_{i \bar{j}}\right)$ if $\omega_{S}=\frac{\sqrt{-1}}{2} g_{i \bar{j}} d z_{i} \wedge d z_{\bar{j}}$.

Theorem 1.1. ( $\left.V=\pi^{*} E, \pi^{*} F_{H_{0}}, X, \omega_{u}\right)$ is a solution of Strominger's system for $\alpha^{\prime}=-2$ if and only if the function $u$ of $S$ satisfies the following Monge-Ampère-type equation:

$$
\triangle e^{u} \cdot \frac{\omega_{S}^{2}}{2 !}+\partial \bar{\partial}\left(e^{-u} \operatorname{tr}\left(\bar{\partial} B \wedge \partial B^{*} \cdot g^{-1}\right)\right)+\partial \bar{\partial} u \wedge \partial \bar{\partial} u=0 .
$$

For the special case, $\omega_{2}=n \omega_{1}, n \in \mathbb{Z},\left(V, \pi^{*} F_{H_{0}}, X, \omega_{u}\right)$ is a solution to Strominger's system if and only if the smooth function $u$ on $S$ satisfies the 
equation:

$$
\triangle\left(e^{u}+\frac{\left(1+n^{2}\right)}{4}\left\|\omega_{1}\right\|_{\omega_{S}}^{2} e^{-u}\right)-8 \frac{\operatorname{det}\left(u_{i \bar{j}}\right)}{\operatorname{det}\left(g_{i \bar{j}}\right)}=0 .
$$

In $[2]$, we proved that

$$
\operatorname{tr}\left(\bar{\partial} B \wedge \partial B^{*} \cdot g^{-1}\right)
$$

is a globally well-defined (1,1)-form on $S$ and when $\omega_{2}=n \omega_{1}, n \in \mathbb{Z}$,

$$
\operatorname{tr}\left(\bar{\partial} B \wedge \partial B^{*} \cdot g^{-1}\right)=\sqrt{-1} \frac{1+n^{2}}{4}\left\|\omega_{1}\right\|_{\omega_{S}}^{2} \omega_{S} .
$$

Let $f=\frac{\left(1+n^{2}\right)}{4}\left\|\omega_{1}\right\|^{2}$ and $g_{i \bar{j}}^{\prime}=\left(e^{u}-f e^{-u}\right) g_{i \bar{j}}-4 u_{i \bar{j}}$, then we can rewrite Equation (1.7) as

$\frac{\operatorname{det} g_{i \bar{j}}^{\prime}}{\operatorname{det} g_{i \bar{j}}}=\left(e^{u}-f e^{-u}\right)^{2}+2\left\{\left(e^{u}+f e^{-u}\right)|\nabla u|^{2}+e^{-u} \Delta f-2 e^{-u} \nabla u \cdot \nabla f\right\}$.

We solve Equation (1.6) by continuity argument [12]. Our main theorem is

Theorem 1.2. There is a smooth solution of Equation (1.6) such that

$$
e^{u} \omega_{S}+\sqrt{-1} e^{-u} \operatorname{tr}\left(\bar{\partial} B \wedge \partial B^{*} \cdot g^{-1}\right)-2 \sqrt{-1} \partial \bar{\partial} u
$$

defines a Hermitian metric on $S$.

Fix a solution $u$ to Equation (1.6). According to Theorem 1.1, $\left(V, F_{\pi^{*} H_{0}}\right.$, $\left.X, \omega_{u}\right)$ defines a reducible solution to Strominger's system. It can be extended to a family of irreducible solution by method of perturbation as was done by $\mathrm{Li}-\mathrm{Yau}[5]$ :

Theorem 1.3. Let $\left(E, H_{0}, S, \omega_{S}\right)$ be as above. Fix the holomorphic structure $D_{0}^{\prime \prime}$ of $E$. Then there is a smooth deformation $D_{s}^{\prime \prime}$ of the holomorphic structure $D_{0}^{\prime \prime}$ on $E$ so that there are Hermitian-Yang-Mills metrics $H_{s}$ on $\left(E, D_{s}\right)$ and smooth functions $\phi_{s}$ on $S$ such that

$$
\left(V=\pi^{*} E, \pi^{*} D_{s}^{\prime \prime}, \pi^{*} H_{s}, \pi^{*}\left(e^{u+\phi_{s}} \omega_{S}\right)+\frac{\sqrt{-1}}{2} \theta \wedge \bar{\theta}\right)
$$

are irreducible solutions to Strominger's system for $\alpha^{\prime}=-2$ on $X$. Furthermore, $\lim _{s \rightarrow 0} \phi_{s}=0$ and $\lim _{s \rightarrow 0} H_{s}$ is a regular reducible Hermitian-YangMills connection on $E=L_{1} \oplus L_{2} \oplus T S$. 


\section{Geometric model}

In this section, we recall the geometric model of Goldstein and Prokushkin

[3] for complex non-Kähler manifolds with $S U(3)$ structure. Their results can be stated as follows:

Theorem 2.1 [3]. Let $\left(S, \omega_{S}, \Omega_{S}\right)$ be a Calabi-Yau surface with a nonvanishing holomorphic $(2,0)$-form $\Omega_{S}$. Let $\omega_{1}$ and $\omega_{2}$ be anti-self-dual $(1,1)$-forms on $S$ such that $\frac{\omega_{1}}{2 \pi}, \frac{\omega_{2}}{2 \pi} \in H^{2}(S, \mathbb{Z})$. Then there is a three-dimensional complex manifold $X$ such that $\pi: X \rightarrow S$ is a holomorphic $T^{2}$-fibration over $S$ with the following properties:

1. For any real 1-forms $\alpha_{1}$ and $\alpha_{2}$ defined on an open subset of $S$ satisfying $d \alpha_{1}=\omega_{1}$ and $d \alpha_{2}=\omega_{2}$, there are local coordinates $x$ and $y$ on $X$ such that $d x+i d y$ defines a holomorphic 1-form on $T^{2}$-fibers and the metric on $X$ has the following form:

$$
g_{0}=\pi^{*} g+\left(d x+\pi^{*} \alpha_{1}\right)^{2}+\left(d y+\pi^{*} \alpha_{2}\right)^{2},
$$

where $g$ is the Ricci-flat metric on $S$.

2. $X$ admits a nowhere vanishing holomorphic (3,0)-form with unit length:

$$
\Omega=\left(\left(d x+\pi^{*} \alpha_{1}\right)+i\left(d y+\pi^{*} \alpha_{2}\right)\right) \wedge \pi^{*} \Omega_{S} .
$$

3. If either $\omega_{1}$ or $\omega_{2}$ represents a non-trivial cohomological class, then $X$ admits no Kähler metric.

4. $X$ is a balanced manifold (see [6]), i.e., the Hermitian form

$$
\omega_{0}=\pi^{*} \omega_{S}+\left(d x+\pi^{*} \alpha_{1}\right) \wedge\left(d y+\pi^{*} \alpha_{2}\right)
$$

satisfies the condition that $d \omega_{0}^{2}=0$.

5. For any smooth function $u$ on $S$, the Hermitian metric

$$
\omega_{u}=\pi^{*}\left(e^{u} \omega_{S}\right)+\left(d x+\pi^{*} \alpha_{1}\right) \wedge\left(d y+\pi^{*} \alpha_{2}\right)
$$

is conformal balanced and $\left(\omega_{u}, \Omega\right)$ satisfies Equation (1.4). 
Let

$$
\begin{aligned}
\theta & =\left(d x+\alpha_{1}\right)+\sqrt{-1}\left(d y+\alpha_{2}\right), \\
\omega_{0} & =\omega_{S}+\frac{\sqrt{-1}}{2} \theta \wedge \bar{\theta}
\end{aligned}
$$

and

$$
\omega_{u}=e^{u} \omega_{S}+\frac{\sqrt{-1}}{2} \theta \wedge \bar{\theta}
$$

Let $R$ and $R_{u}$ be the curvature forms of the Hermitian connections on the holomorphic tangent bundle corresponding to Hermitian metrics $\omega_{0}$ and $\omega_{u}$, respectively. Then, we have

Proposition 2.2 [2].

$$
\operatorname{tr} R \wedge R=\pi^{*}\left(\operatorname{tr} R_{S} \wedge R_{S}+2 \operatorname{tr} \partial \bar{\partial}\left(\bar{\partial} B \wedge \partial B^{*} \cdot g^{-1}\right)\right) .
$$

Proposition 2.3 [2].

$$
\begin{aligned}
\operatorname{tr} R_{u} \wedge R_{u}= & \pi^{*}\left(\operatorname{tr} R_{S} \wedge R_{S}+2 \partial \bar{\partial}\left(e^{-u} \operatorname{tr}\left(\bar{\partial} B \wedge \partial B^{*} \cdot g^{-1}\right)\right)\right. \\
& +2 \partial \bar{\partial} u \wedge \partial \bar{\partial} u) .
\end{aligned}
$$

Proposition $2.4[2]$. When $\omega_{2}=n \omega_{1}, n \in \mathbb{Z}$,

$$
\operatorname{tr}\left(\bar{\partial} B \wedge \partial B^{*} \cdot g^{-1}\right)=\frac{\sqrt{-1}}{4}\left(1+n^{2}\right)\left\|\omega_{1}\right\|_{\omega_{S}}^{2} \omega_{S}
$$

The Laplace operator $\triangle$ on $S$ is defined by $\triangle=\bar{\partial}^{*} \circ \bar{\partial}$, where $\bar{\partial}^{*}$ is defined by the metric $\omega_{S}$. For any smooth function $\psi$ on $S$, we have

$$
\sqrt{-1} \partial \bar{\partial} \psi \wedge \omega_{S}=\triangle \psi \cdot \frac{\omega_{S}^{2}}{2 !}
$$

Lemma $2.5[2]$.

$$
\sqrt{-1} \partial \bar{\partial} \omega_{u}=\triangle e^{u} \cdot \frac{\omega_{S}^{2}}{2 !}+\frac{1}{2}\left(\left\|\omega_{1}\right\|_{\omega_{S}}^{2}+\left\|\omega_{2}\right\|_{\omega_{S}}^{2}\right) \frac{\omega_{S}^{2}}{2 !} .
$$

\section{Construction of solution to Strominger's system with reducible bundle}

In this section, we demonstrate the existence of smooth solutions to Strominger's system. As $\frac{\omega_{1}}{2 \pi}, \frac{\omega_{2}}{2 \pi} \in H^{1,1}(S, \mathbb{R}) \cap H^{2}(S, \mathbb{Z})$, there are holomorphic line bundles $L_{1}$ and $L_{2}$ over $S$ such that the curvature forms of their 
Hermitian connections are $\sqrt{-1} \omega_{1}$ and $\sqrt{-1} \omega_{2}$, respectively (see [10]). Associated to these curvature forms, there exist Hermitian metrics $h_{1}$ and $h_{2}$ on $L_{1}$ and $L_{2}$. Let

$$
E=L_{1} \oplus L_{2} \oplus T^{\prime} S
$$

and

$$
H_{0}=\left(h_{1}, h_{2}, \omega_{S}\right) .
$$

The curvature form $F_{H_{0}}$ of $E$ is given by

$$
F_{H_{0}}=\left(\begin{array}{ccc}
\sqrt{-1} \omega_{1} & & \\
& \sqrt{-1} \omega_{2} & \\
& & R_{S}
\end{array}\right),
$$

where $R_{S}$ is the curvature of $T^{\prime} S$ associate to the Hermitian metric $\omega_{S}$. Let $V=\pi^{*} E$ and $F_{\tilde{H}_{0}}=\pi^{*} F_{H_{0}}$.

Lemma 3.1. For any smooth function $u$ on $S,\left(V, F_{\tilde{H}_{0}}, X, \omega_{u}\right)$ satisfies Equations (1.1), (1.2) and (1.4).

Proof. By Theorem 2.1, Equation (1.4) is satisfied. As $F_{\tilde{H}_{0}}$ is the (1,1)-form on $X, F_{\tilde{H}_{0}}^{2,0}=F_{\tilde{H}_{0}}^{0,2}=0$. We also have

$$
F_{\tilde{H}_{0}} \wedge \omega_{u}^{2}=\pi^{*}\left(F_{H_{0}} \wedge e^{u} \omega_{S}\right) \wedge\left(\pi^{*}\left(e^{u} \omega_{S}\right)+\sqrt{-1} \theta \wedge \bar{\theta}\right)=0
$$

as follows from the equation $\omega_{1} \wedge \omega_{S}=\omega_{2} \wedge \omega_{S}=0$ and $R_{S} \wedge \omega_{S}=0$.

It remains to consider Equation (1.3). We normalize $\alpha^{\prime}=-2$, then $\left(V, F_{\tilde{H}_{0}}, X, \omega_{u}\right)$ satisfies the equation

$$
\sqrt{-1} \partial \bar{\partial} \omega_{u}=\frac{1}{2}\left(\operatorname{tr} F_{\tilde{H}_{0}} \wedge F_{\tilde{H}_{0}}-\operatorname{tr} R_{u} \wedge R_{u}\right)
$$

and

$$
\begin{aligned}
\operatorname{tr} F_{\tilde{H}_{0}} \wedge F_{\tilde{H}_{0}} & =-\omega_{1} \wedge \omega_{1}-\omega_{2} \wedge \omega_{2}+\operatorname{tr} R_{S} \wedge R_{S} \\
& =\left(\left\|\omega_{1}\right\|^{2}+\left\|\omega_{2}\right\|^{2}\right) \frac{\omega_{S}^{2}}{2 !}+\operatorname{tr} R_{S} \wedge R_{S} .
\end{aligned}
$$

Inserting (2.6), (2.9) and (3.4) into (3.3), we can rewrite Equation (3.3) as

$$
\triangle e^{u} \cdot \frac{\omega_{S}^{2}}{2 !}+\partial \bar{\partial}\left(e^{-u} \operatorname{tr}\left(\bar{\partial} B \wedge \partial B^{*} \cdot g^{-1}\right)\right)+\partial \bar{\partial} u \wedge \partial \bar{\partial} u=0 .
$$

In conclusion, we have 
Theorem 3.2. $\left(V, F_{\pi^{*} H_{0}}, X, \omega_{u}\right)$ is a solution of Strominger's system for $\alpha^{\prime}=-2$ if and only if the function $u$ of $S$ satisfies Equation (3.5). In particular, when $\omega_{2}=n \omega_{1}, n \in \mathbb{Z},\left(V, F_{\pi^{*} H_{0}}, X, \omega_{u}\right)$ is a solution to Strominger's system if and only if the smooth function $u$ on $S$ satisfies the equation:

$$
\triangle\left(e^{u}+\frac{\left(1+n^{2}\right)}{4}\left\|\omega_{1}\right\|_{\omega_{S}}^{2} e^{-u}\right) \frac{\omega_{S}^{2}}{2 !}+\partial \bar{\partial} u \wedge \partial \bar{\partial} u=0
$$

or

$$
\triangle\left(e^{u}+\frac{\left(1+n^{2}\right)}{4}\left\|\omega_{1}\right\|_{\omega_{S}}^{2} e^{-u}\right)-8 \frac{\operatorname{det}\left(u_{i \bar{j}}\right)}{\operatorname{det}\left(g_{i \bar{j}}\right)}=0
$$

Proof. For the second part of theorem, we note that when $\omega_{2}=n \omega_{1}$, it follows from Proposition 2.4 and (2.8) that

$$
\begin{aligned}
\partial \bar{\partial}\left(e^{-u} \operatorname{tr}\left(\bar{\partial} B \wedge \partial B^{*} \cdot g^{-1}\right)\right) & =\sqrt{-1} \frac{\left(1+n^{2}\right)}{4} \partial \bar{\partial}\left(\left\|\omega_{1}\right\|_{\omega_{S}}^{2} e^{-u}\right) \wedge \omega_{S} \\
& =\frac{\left(1+n^{2}\right)}{4} \triangle\left(\left\|\omega_{1}\right\|_{\omega_{S}}^{2} e^{-u}\right) \frac{\omega_{S}^{2}}{2 !} .
\end{aligned}
$$

So, Equation (3.6) follows from (3.5). Equation (3.7) is derived from

$$
\begin{aligned}
\partial \bar{\partial} u \wedge \partial \bar{\partial} u & =2 \operatorname{det}\left(u_{i \bar{j}}\right) d z_{1} \wedge d \bar{z}_{1} \wedge d z_{2} \wedge d \bar{z}_{2} \\
& =-8 \frac{\operatorname{det}\left(u_{i \bar{j}}\right)}{\operatorname{det}\left(g_{i \bar{j}}\right)} \frac{\omega_{S}^{2}}{2 !} .
\end{aligned}
$$

Theorem 3.3. There is a smooth solution of Equation (3.5) such that

$$
e^{u} \omega_{S}+\sqrt{-1} e^{-u} \operatorname{tr}\left(\bar{\partial} B \wedge \partial B^{*} \cdot g^{-1}\right)-2 \sqrt{-1} \partial \bar{\partial} u
$$

defines a Hermitian metric on $S$.

Proof. We solve Equation (3.5) by the continuity method. More precisely, we introduce a parameter $t \in[0,1]$ and consider the following equation

$$
\sqrt{-1} \partial \bar{\partial} e^{u} \wedge \omega_{S}+t \partial \bar{\partial}\left(e^{-u} \operatorname{tr}\left(\bar{\partial} B \wedge \partial B^{*} \cdot g^{-1}\right)\right)+\partial \bar{\partial} u \wedge \partial \bar{\partial} u=0 .
$$

We shall impose the following:

$$
\text { Elliptic condition : } \begin{aligned}
\omega^{\prime}= & e^{u} \omega_{S}+\sqrt{-1} t e^{-u} \operatorname{tr}\left(\bar{\partial} B \wedge \partial B^{*} \cdot g^{-1}\right) \\
& -2 \sqrt{-1} \partial \bar{\partial} u>0
\end{aligned}
$$


and

$$
\text { Normalization condition : } \int_{S} e^{-u} \frac{\omega_{S}^{2}}{2 !}=A, \quad \int_{S} 1 \frac{\omega_{S}^{2}}{2 !}=1 \text {. }
$$

Let $C^{k, \alpha}(S)$ be the space of functions whose $k t h$ derivatives are Hölder continuous with exponent $0 \leq \alpha \leq 1$. We consider the solution in the following space

$$
B_{A}=\left\{u \in C^{2, \alpha}(S) \mid u \text { satisfies the normalization (3.10) }\right\}
$$

and

$$
B_{A, t}=\left\{u \in B_{A} \mid u \text { also satisfies the elliptic condition (3.9) }\right\}
$$

Let

$$
\mathbf{T}=\left\{t \in[0,1] \mid \text { Equation (3.5) admits a solution in } B_{A, t}\right\}
$$

Obviously $0 \in \mathbf{T}$ with a solution $u=-\ln A$. Hence, we only need to show that $\mathbf{T}$ is both closed and open in $[0,1]$. This will imply that $1 \in \mathbf{T}$ and that our original equation has a solution in $C^{2, \alpha}$. By the Schauder theory, this solution is smooth. The proof of openness is the same as the proof of our Theorem 18 in [2].

So, we need only to prove that $\mathbf{T}$ is closed. We need to estimate up to third order. The third-order estimate is the same as in our previous paper [2]. Hence we shall provide estimates up to second order. These will be done in Section 4, 5 and 6. In Section 7, we shall explain why the estimates to Equation (3.7) can be generalized to Equation (3.5).

\section{Zero-order estimate}

Let $f=\frac{1+n^{2}}{4}\left\|\omega_{1}\right\|^{2}$, where $\omega_{1}$ is the anti-self dual $(1,1)$-form on $S$. Then Equation (3.7) can be written as

$$
\triangle\left(e^{u}+f e^{-u}\right)-8 \frac{\operatorname{det}\left(u_{i \bar{j}}\right)}{\operatorname{det}\left(g_{i \bar{j}}\right)}=0 .
$$

The elliptic condition says

$$
\omega^{\prime}=\left(e^{u}-t f e^{-u}\right) \omega_{S}-2 \sqrt{-1} \partial \bar{\partial} u>0
$$


and the normalization is

$$
\int_{S} e^{-u} \frac{\omega_{S}^{2}}{2 !}=A, \quad \int_{S} 1 \frac{\omega_{S}^{2}}{2 !}=1
$$

In the following, the volume form will be $\frac{\omega_{S}^{2}}{2 !}$ unless it is clear from the context. Multiplying the elliptic condition $e^{u}-f e^{-u}>\Delta u$ by $p e^{-p u}$, we obtain

$$
p\left(e^{-p u}\right)\left(e^{u}-f e^{-u}\right) \geq p\left(e^{-p u}\right) \triangle u=-\triangle\left(e^{-p u}\right)+4\left|\nabla\left(e^{-u}\right)^{p / 2}\right|^{2}
$$

and by integrating, we obtain

$$
\int_{S}\left|\nabla\left(e^{-u}\right)^{p / 2}\right|^{2} \leq \frac{p}{4} \int_{S}\left(e^{-u}\right)^{p-1}
$$

Applying the Sobolev inequality, we can find a constant $C$ depending only on $S$ such that

$$
\begin{aligned}
\left(\int_{S}\left(e^{-u}\right)^{2 p}\right)^{1 / 2} & \leq C \int_{S}\left(e^{-u}\right)^{p}+C \int_{S}\left|\nabla\left(e^{-u}\right)^{p / 2}\right|^{2} \\
& \leq C \int_{S}\left(e^{-u}\right)^{p}+\frac{p}{4} C \int_{S}\left(e^{-u}\right)^{p-1} .
\end{aligned}
$$

In the following, we use the constant in the generic sense. So $C$ may mean different constants in different equations. But it shall be independent of $u$. By (4.5) and the Hölder inequality, we find

$$
\begin{aligned}
\int_{S}\left(e^{-u}\right)^{2 p} & \leq C^{2}\left(\int_{S}\left(e^{-u}\right)^{p}\right)^{2}+C^{2} p^{2}\left(\int_{S}\left(e^{-u}\right)^{p-1}\right)^{2} \\
& \leq C^{2}\left(\int_{S}\left(e^{-u}\right)^{p}\right)^{2}+C^{2} p^{2}\left(\int_{S}\left(e^{-u}\right)^{p}\right)^{2(p-1) / p} .
\end{aligned}
$$

We assume that

$$
\int_{S} e^{-u}=A<1
$$


There are two cases:

Case (1). For any $p \in \mathbb{Z}, \int_{S} e^{-p u}<1 . \quad$ Then, $\left(\int_{S}\left(e^{-u}\right)^{p}\right)^{2}<\left(\int_{S}\right.$ $\left.\left(e^{-u}\right)^{p}\right)^{2(p-1) / p}$ and from (4.6),

$$
\int_{S}\left(e^{-u}\right)^{2 p} \leq C^{2} p^{2}\left(\int_{S}\left(e^{-u}\right)^{p}\right)^{2(p-1) / p} .
$$

Let $2 p=2^{\beta}$, then

$$
\begin{aligned}
\int_{S}\left(e^{-u}\right)^{2^{\beta}} & \leq C^{2}\left(2^{\beta-1}\right)^{2}\left(\int_{S}\left(e^{-u}\right)^{2^{\beta-1}}\right)^{2\left(1-2^{-(\beta-1)}\right)} \\
& \leq\left(\prod_{b=1}^{\beta-1} C^{2^{b}}\right)\left(\prod_{b=1}^{\beta-1}\left(2^{(\beta-b)}\right)^{2^{b}}\right)\left(\int_{S}\left(e^{-u}\right)^{2}\right)^{2^{\beta-1} \cdot \prod_{k=1}^{\beta-1}\left(1-1 / 2^{k}\right)} \\
& \leq C^{2^{\beta}-2} \cdot 2^{2^{\beta+1}}\left(\int_{S}\left(e^{-u}\right)^{2}\right)^{2^{\beta-1} \cdot \prod_{k=1}^{\beta-1}\left(1-1 / 2^{k}\right)}
\end{aligned}
$$

where the last inequality follows from

$$
\prod_{b=1}^{\beta-1}\left(2^{\beta-b}\right)^{2^{b}} \leq 2^{2^{\beta+1}}
$$

which can be derived from the following calculation:

$$
\begin{aligned}
\prod_{b=1}^{\beta-1}\left(2^{\beta-b}\right)^{2^{b}} & =\prod_{b=1}^{\beta-1} 2^{2^{b}} \prod_{b=1}^{\beta-1}\left(2^{\beta-(b+1)}\right)^{2^{b}} \\
& =2^{2^{\beta}-2}\left(\prod_{b=1}^{\beta-1}\left(2^{\beta-(b+1)}\right)^{2^{b+1}}\right)^{1 / 2} \\
& =2^{2^{\beta}-1-\beta}\left(\prod_{b=1}^{\beta-1}\left(2^{\beta-b}\right)^{2^{b}}\right)^{1 / 2} \\
& \leq 2^{2^{\beta}}\left(\prod_{b=1}^{\beta-1}\left(2^{\beta-b}\right)^{2^{b}}\right)^{1 / 2} .
\end{aligned}
$$

Hence, we obtain

$$
\left(\int\left(e^{-u}\right)^{2^{\beta}}\right)^{1 / 2^{\beta}} \leq C^{1-2^{1-\beta}} \cdot 2^{2}\left(\int_{S}\left(e^{-u}\right)^{2}\right)^{1 / 2 \cdot \prod_{k=1}^{\beta-1}\left(1-1 / 2^{k}\right)} .
$$


Let $\beta \rightarrow \infty$, we see that

$$
\exp (-\inf u)=\left\|e^{-u}\right\|_{\infty} \leq C\left(\int_{S}\left(e^{-u}\right)^{2}\right)^{\kappa / 2}
$$

where

$$
\kappa=\prod_{\beta=1}^{\infty}\left(1-\frac{1}{2^{\beta}}\right)>0 .
$$

To finish our estimate of $\inf u$, it suffices to estimate $\left\|e^{-u}\right\|_{2}$. When $p=2$, inequality (4.4) yields

$$
\int_{S}\left|\nabla\left(e^{-u}\right)\right|^{2} \leq \frac{1}{2} \int_{S} e^{-u}
$$

Now from the normalization condition (4.3), we have $\int_{S}\left(e^{-u}-A\right)=0$. Poincare inequality and (4.11) then imply

$$
\int_{S}\left|\left(e^{-u}-A\right)\right|^{2} \leq C \int_{S}\left|\nabla\left(e^{-u}-A\right)\right|^{2} \leq C A
$$

and

$$
\int_{S}\left(e^{-u}\right)^{2} \leq A^{2}+C A \leq C A .
$$

Combining (4.9) and (4.12), we get

$$
\exp (-\inf u)=\left\|e^{-u}\right\|_{\infty} \leq C_{1} A^{\kappa / 2}
$$

and

$$
\inf u \geq-\ln C_{1}-\frac{\kappa}{2} \ln A \text {. }
$$

Case (2). There is an integer $p$ such that $\int_{S} e^{-p u}>1$. Let $p_{0}$ be the first such integer. Then, for any $p>p_{0}$, the Hölder inequality implies that

$$
\int_{S} e^{-p u} \geq\left(\int_{S} e^{-p_{0} u}\right)^{p / p_{0}}>1
$$

From (4.5),

$$
\int_{S}\left(e^{-u}\right)^{2 p} \leq C^{2} p^{2}\left(\int_{S}\left(e^{-u}\right)^{p}\right)^{2} \quad \text { for } p \geq p_{0}
$$


and

$$
\int_{S}\left(e^{-u}\right)^{2 p} \leq C^{2} p^{2}\left(\int_{S}\left(e^{-u}\right)^{p}\right)^{2(p-1) / p} \text { for } p<p_{0} .
$$

From these two inequalities, as was discussed in case (1), we obtain the estimate of $\inf u$. Furthermore, we have bound (4.14) with the same $\kappa$ satisfying (4.10).

Next, we estimate $\sup _{S} u$. Let $g^{\prime}=\frac{\sqrt{-1}}{2} g_{i \bar{j}}^{\prime} d z_{i} \wedge d z_{\bar{j}}$, where

$$
g_{i \bar{j}}^{\prime}=\left(e^{u}-f e^{-u}\right) g_{i \bar{j}}-4 \alpha u_{i \bar{j}}
$$

We note that

$$
\frac{\omega^{\prime 2}}{2 !}=\frac{\operatorname{det} g_{i \bar{j}}^{\prime}}{\operatorname{det} g_{i \bar{j}}} \frac{\omega_{S}^{2}}{2 !}
$$

The matrix $\left(g^{\prime i \bar{j}}\right)$ satisfies the equation

$$
\sum g^{\prime i \bar{j}} g_{i \bar{j}}=\delta_{\bar{k}}^{\bar{j}}
$$

So,

$$
g^{\prime 1 \overline{1}}=\frac{g_{2 \overline{2}}^{\prime}}{\operatorname{det} g_{i \bar{j}}^{\prime}}, \quad g^{\prime 1 \overline{2}}=-\frac{g_{2 \overline{1}}^{\prime}}{\operatorname{det} g_{i \bar{j}}^{\prime}}, \quad g^{\prime 2 \overline{1}}=-\frac{g_{1 \overline{2}}^{\prime}}{\operatorname{det} g_{i \bar{j}}^{\prime}}, \quad g_{2 \overline{2}}^{\prime}=\frac{g_{1 \overline{1}}^{\prime}}{\operatorname{det} g_{i \bar{j}}^{\prime}} .
$$

We define the operator

$$
P(\varphi) \frac{\omega^{\prime 2}}{2 !}=\sqrt{-1} \partial \bar{\partial} \varphi \wedge \omega^{\prime}
$$

so that $P(\varphi)=2 g^{\prime i \bar{j}} \varphi_{i \bar{j}}$. By computation,

$$
\begin{aligned}
& \int_{S} P\left(e^{k u}\right) \frac{\operatorname{det} g^{\prime}}{\operatorname{det} g} \frac{\omega^{2}}{2 !} \\
& =\int_{S} 2 g^{\prime i \bar{j}} \frac{\partial^{2}\left(e^{k u}\right)}{\partial z_{i} \partial \bar{z}_{j}} \frac{\operatorname{det} g^{\prime}}{\operatorname{det} g} \frac{\omega^{2}}{2 !} \\
& =2 k^{2} \int_{S} g^{\prime i \bar{j}} e^{k u} \frac{\partial u}{\partial z_{i}} \frac{\partial u}{\partial \bar{z}_{j}} \frac{\omega^{2}}{2 !}+2 k \int_{S} g^{\prime i \bar{j}} e^{k u} \frac{\partial^{2} u}{\partial z_{i} \partial \bar{z}_{j}} \frac{\operatorname{det} g^{\prime}}{\operatorname{det} g} \frac{\omega^{2}}{2 !} \\
& \geq k \int_{S} e^{k u}\left(2 g^{\prime i \bar{j}} \frac{\partial^{2} u}{\partial z_{i} \partial \bar{z}_{j}} \frac{\operatorname{det} g^{\prime}}{\operatorname{det} g}\right) \frac{\omega^{2}}{2 !} .
\end{aligned}
$$


Applying the equation, we find

$$
\begin{aligned}
2 g^{\prime i \bar{j}} \frac{\partial^{2} u}{\partial z_{i} \partial \bar{z}_{j}} \frac{\operatorname{det} g^{\prime}}{\operatorname{det} g}= & \left(2 g_{2 \overline{2}}^{\prime} \frac{\partial^{2} u}{\partial z_{1} \partial \bar{z}_{1}}+2 g_{1 \overline{1}}^{\prime} \frac{\partial^{2} u}{\partial z_{2} \partial \bar{z}_{2}}\right. \\
& \left.-2 g_{1 \overline{2}}^{\prime} \frac{\partial^{2} u}{\partial z_{2} \partial \bar{z}_{1}}-2 g_{2 \overline{1}}^{\prime} \frac{\partial^{2} u}{\partial z_{1} \partial \bar{z}_{2}}\right) \frac{1}{\operatorname{det} g} \\
= & 2\left(e^{u}-f e^{-u}\right) g^{i \bar{j}} \frac{\partial^{2} u}{\partial z_{i} \partial \bar{z}_{j}}-16 \frac{\operatorname{det} u_{i \bar{j}}}{\operatorname{det} g} \\
= & \left(e^{u}-f e^{-u}\right) \triangle u-2 \triangle\left(e^{u}+f e^{-u}\right) .
\end{aligned}
$$

Inserting (4.16) into (4.15), we get

$$
\begin{aligned}
\int_{S} P\left(e^{k u}\right) \frac{\operatorname{det} g^{\prime}}{\operatorname{det} g} \frac{\omega^{2}}{2 !} \geq & k \int_{S} e^{k u}\left(e^{u}-f e^{-u}\right) \triangle u \frac{\omega^{2}}{2 !} \\
& -2 k \int_{S} e^{k u} \triangle\left(e^{u}+f e^{-u}\right) \frac{\omega^{2}}{2 !}
\end{aligned}
$$

On the other hand, from the definition of the operator $P$, we have

$$
\begin{aligned}
\int_{S} P\left(e^{k u}\right) \frac{\operatorname{det} g^{\prime}}{\operatorname{det} g} \frac{\omega^{2}}{2 !}= & \int_{S} P\left(e^{k u}\right) \frac{\omega^{\prime 2}}{2 !}=\int_{S} \sqrt{-1} \partial \bar{\partial}\left(e^{k u}\right) \wedge \omega^{\prime} \\
= & \int_{S} \sqrt{-1} \partial \bar{\partial}\left(e^{k u}\right) \\
& \wedge\left(\left(e^{u}-f e^{-u}\right) \omega-2 \sqrt{-1} \partial \bar{\partial} u\right) \\
= & \int_{S}\left(e^{u}-f e^{-u}\right) \triangle\left(e^{k u}\right) \frac{\omega^{2}}{2 !} \\
& +2 \int_{S} \partial \bar{\partial}\left(e^{k u}\right) \wedge \partial \bar{\partial} u \\
= & k \int_{S} e^{k u}\left(e^{u}-f e^{-u}\right) \triangle u \frac{\omega^{2}}{2 !} \\
& +k^{2} \int_{S}\left(e^{u}-f e^{-u}\right) e^{k u}|\nabla u|^{2} \frac{\omega^{2}}{2 !}
\end{aligned}
$$


where $|\nabla u|^{2}=2 g^{i \bar{j}} \partial_{i} u \partial_{\bar{j}} u$. Combining (4.17) and (4.18), we see that

$$
\begin{aligned}
& k^{2} \int_{S}\left(e^{u}-f e^{-u}\right) e^{k u}|\nabla u|^{2} \frac{\omega^{2}}{2 !} \\
& \geq-2 k \int_{S} e^{k u} \triangle\left(e^{u}+f e^{-u}\right) \frac{\omega^{2}}{2 !} \\
& =-2 k \int_{S} e^{k u}\left(e^{u}-f e^{-u}\right) \triangle u \frac{\omega^{2}}{2 !}-2 k \int_{S} e^{k u}\left(e^{u}+f e^{-u}\right)|\nabla u|^{2} \\
& -2 k \int_{S} e^{(k-1) u} \triangle f+4 k \int_{S} e^{(k-1) u} \nabla u \cdot \nabla f .
\end{aligned}
$$

Integration by part, we find

$$
\begin{aligned}
- & 2 k \int_{S} e^{k u}\left(e^{u}-f e^{-u}\right)(\triangle u) \frac{\omega^{2}}{2 !} \\
& =2 k(k+1) \int_{S} e^{(k+1) u}|\nabla u|^{2}-2 k(k-1) \int_{S} f e^{(k-1) u}|\nabla u|^{2} \\
& -\frac{2 k}{k-1} \int_{S} e^{(k-1) u} \triangle f-4 k \int_{S} e^{(k-1) u} \nabla u \cdot \nabla f .
\end{aligned}
$$

Inserting (4.20) into (4.19) and applying Schwarz' inequality, we get

$$
\begin{aligned}
& k^{2} \int_{S}\left(e^{u}-f e^{-u}\right) e^{k u}|\nabla u|^{2} \frac{\omega^{2}}{2 !} \\
& \quad \geq 2 k^{2} \int_{S} e^{(k+1) u}|\nabla u|^{2} \\
& \quad-2 k^{2} \int_{S} e^{(k-1) u} f|\nabla u|^{2}-2 k\left(1+\frac{1}{k-1}\right) \int_{S} e^{(k-1) u} \Delta f
\end{aligned}
$$

and

$$
\begin{aligned}
& 2 k\left(1+\frac{1}{k-1}\right) \int_{S} e^{(k-1) u} \triangle f \\
& \quad \geq k^{2} \int_{S} e^{(k+1) u}|\nabla u|^{2}-k^{2} \int_{S} e^{(k-1) u} f|\nabla u|^{2} .
\end{aligned}
$$

If we take $A>0$ small enough such that

$$
C_{1}^{-1} A^{-\kappa / 2}>1+\sup f
$$


then from (4.13) we see that inf $e^{u}>1+\sup f$ and

$$
\begin{aligned}
& k^{2} \int_{S} e^{(k+1) u}|\nabla u|^{2}-k^{2} \int_{S} e^{(k-1) u} f|\nabla u|^{2} \\
& \quad \geq C k^{2} \int_{S} e^{(k+1) u}|\nabla u|^{2}=\frac{4 C k^{2}}{(k+1)^{2}} \int_{S}\left|\nabla\left(e^{u}\right)^{(k+1) / 2}\right|^{2} .
\end{aligned}
$$

Equations (4.21) and (4.23) imply that for all $k \geq 1$,

$$
\int_{S}\left|\nabla\left(e^{u}\right)^{(k+1) / 2}\right|^{2} \leq C(k+1) \int_{S} e^{(k-1) u} .
$$

Now applying the Sobolev inequality and $\inf u>0$, we get

$$
\begin{aligned}
\left(\int\left(e^{u}\right)^{2 k}\right)^{1 / 2} & \leq C \int\left(e^{u}\right)^{k}+C \int\left|\nabla\left(e^{u}\right)^{k / 2}\right|^{2} \\
& \leq C \int\left(e^{u}\right)^{k}+C k \int\left(e^{u}\right)^{k-2} \leq C k \int_{S}\left(e^{u}\right)^{k}
\end{aligned}
$$

Take $2 k=2^{\beta}$. Equation (4.25) implies

$$
\begin{aligned}
\int_{S}\left(e^{u}\right)^{2^{\beta}} & \leq C\left(2^{\beta-1}\right)^{2}\left(\int_{S}\left(e^{u}\right)^{2^{\beta-1}}\right)^{2} \\
& \leq C^{2^{\beta}-2} \prod_{b=1}^{\beta-1}\left(2^{\beta-b}\right)^{2^{b}}\left(\int_{S}\left(e^{u}\right)^{2}\right)^{2^{\beta-1}} \\
& \leq C^{2^{\beta}-2} 2^{2^{\beta+1}}\left(\int_{S}\left(e^{u}\right)^{2}\right)^{2^{\beta-1}}
\end{aligned}
$$

where the last inequality follows from (4.8). Hence,

$$
\left(\int_{S}\left(e^{u}\right)^{2^{\beta}}\right)^{1 / 2^{\beta}} \leq C\left(\int_{S}\left(e^{u}\right)^{2}\right)^{1 / 2} .
$$

Let $\beta \rightarrow \infty$, then we get

$$
\sup u=\|u\|_{\infty}=C\left(\int_{S}\left(e^{u}\right)^{2}\right)^{1 / 2} .
$$

Now we estimate $\left\|e^{u}\right\|_{2}$. When $k=1$, inequality (4.24) yields

$$
\int_{S}\left|\nabla\left(e^{u}\right)\right|^{2} \leq C
$$


Let $M_{u}=\int_{S} e^{u}$, then $\int_{S}\left(e^{u}-M_{u}\right)=0$. Applying the Poincare inequality and (4.27), we get

$$
\begin{aligned}
\int_{S}\left(e^{u}\right)^{2}-\left(\int_{S}\left(e^{u}\right)\right)^{2} & =\int_{S}\left(e^{u}-M_{u}\right)^{2} \\
& \leq C \int_{S}\left|\nabla\left(e^{u}-M_{u}\right)\right|^{2} \\
& =C \int_{S}\left|\nabla e^{u}\right|^{2} \leq C .
\end{aligned}
$$

Hence, there is a constant $C_{2}$ depending on $S, f$ and $A$ (recall (4.22)) such that

$$
\int_{S}\left(e^{u}\right)^{2} \leq\left(\int_{S}\left(e^{u}\right)\right)^{2}+C_{2} .
$$

Let $U_{1}=\left\{x \in S \mid \exp (-u(x)) \geq \frac{A}{2}\right\} \quad$ and $\quad U_{2}=\left\{x \in S \mid \exp (-u(x))<\frac{A}{2}\right\}$. Then, from (4.13), we have

$$
\begin{aligned}
A & =\int_{S} e^{-u}=\int_{U_{1}} e^{-u}+\int_{U_{2}} e^{-u}<\int_{U_{1}} e^{-\inf u}+\int_{U_{2}} \frac{A}{2} \\
& =e^{-\inf u} \operatorname{Vol}\left(U_{1}\right)+\frac{A}{2}\left(1-\operatorname{Vol}\left(U_{2}\right)\right) \\
& \leq\left(C_{1} A^{\kappa / 2}-\frac{A}{2}\right) \operatorname{Vol}\left(U_{1}\right)+\frac{A}{2} .
\end{aligned}
$$

As $0<\kappa<1$ and $0<A<1$, we can choose $A$ small enough such that

$$
A<\left(2 C_{1}\right)^{1 /(1-\kappa) / 2} \text {. }
$$

Then,

$$
\operatorname{Vol}\left(U_{1}\right) \geq \frac{A / 2}{C_{1} A^{\kappa / 2}-A / 2}=\frac{1}{2 C_{1} A^{\kappa / 2-1}-1}>0
$$

and hence

$$
\operatorname{Vol}\left(U_{2}\right)=\left(1-\operatorname{Vol}\left(U_{1}\right)\right)<1-\frac{1}{2 C_{1} A^{\kappa / 2-1}-1}<1
$$


Now we want to use (4.28) and (4.30) to estimate $\left\|e^{u}\right\|_{2}$. Applying Young's inequality and the Hölder inequality, we find

$$
\begin{aligned}
\left(\int_{S} e^{u}\right)^{2}= & \left(\int_{U_{1}} e^{u}+\int_{U_{2}} e^{u}\right)^{2} \\
\leq & \left(1+\frac{1}{\epsilon}\right)\left(\int_{U_{1}} e^{u}\right)^{2}+(1+\epsilon)\left(\int_{U_{2}} e^{u}\right)^{2} \\
\leq & \left(1+\frac{1}{\epsilon}\right)\left(\int_{U_{1}} e^{2 u}\right) \operatorname{Vol}\left(U_{1}\right) \\
& +(1+\epsilon) \operatorname{Vol}\left(U_{2}\right)\left(\int_{U_{2}} e^{2 u}\right) \\
\leq & \left(1+\frac{1}{\epsilon}\right)\left(\frac{2}{A}\right)^{2}+(1+\epsilon) \operatorname{Vol}\left(U_{2}\right)\left(\int_{U_{2}} e^{2 u}\right) \\
\leq & \left(1+\frac{1}{\epsilon}\right)\left(\frac{2}{A}\right)^{2}+(1+\epsilon) \operatorname{Vol}\left(U_{2}\right)\left(\int_{S} e^{2 u}\right) .
\end{aligned}
$$

Inserting (4.28) and (4.30) into (4.31),

$$
\begin{aligned}
\left(\int_{S} e^{u}\right)^{2} \leq & \left(1+\frac{1}{\epsilon}\right)\left(\frac{2}{A}\right)^{2}+C_{2}(1+\epsilon)\left(1-\frac{1}{2 C_{1} A^{\frac{\kappa}{2}-1}-1}\right) \\
& +(1+\epsilon)\left(1-\frac{1}{2 C_{1} A^{\frac{\kappa}{2}-1}-1}\right)\left(\int_{S} e^{u}\right)^{2} .
\end{aligned}
$$

Taking $\epsilon$ small enough such that

$$
(1+\epsilon)\left(1-\frac{1}{2 C_{1} A^{\kappa / 2-1}-1}\right)<1,
$$

then we get

$$
\left(\int_{S} e^{u}\right)^{2}<\frac{(1+1 / \epsilon)(2 / A)^{2}+C_{2}(1+\epsilon)\left(1-1 /\left(2 C_{1} A^{\kappa / 2-1}-1\right)\right)}{1-(1+\epsilon)\left(1-1 /\left(2 C_{1} A^{\kappa / 2-1}-1\right)\right)} .
$$

Now estimate of $\int_{S}\left(e^{u}\right)^{2}$ follows from (4.28) and the estimate of $\sup u$ follows from (4.26).

\section{Gradient estimate}

Suppose the function $\ln |\nabla u|^{2}+\ln v(u)$ achieves its maximum at the point $q_{1} \in S$, where $v$ is a positive function of $u$ to be determined. Then, at $q_{1}$, 
we have

$$
\nabla\left(|\nabla u|^{2}\right)=-\left(\frac{v^{\prime}(u)}{v(u)}|\nabla u|^{2}\right) \nabla u
$$

We may choose the normal coordinate at $q_{1}$ such that $\frac{\partial u}{\partial z_{1}} \neq 0$ and $\frac{\partial u}{\partial z_{2}}=0$. As $u$ is real, we can assume that $\frac{\partial u}{\partial x_{1}}>0$ and $\frac{\partial u}{\partial y_{1}}=0$. Thus, we can assume that at $q_{1}$,

$$
\partial_{1} u \partial_{\overline{1}} u=\partial_{1} u \partial_{1} u=\partial_{\overline{1}} u \partial_{\overline{1}} u=\frac{1}{2}|\nabla u|^{2} .
$$

From (5.1), we get

$$
\partial_{1} \partial_{1} u+\partial_{1} \partial_{\overline{1}} u=\partial_{\overline{1}} \partial_{\overline{1}} u+\partial_{1} \partial_{\overline{1}} u=-\frac{1}{2} \frac{v^{\prime}(u)}{v(u)}|\nabla u|^{2}
$$

and

$$
\partial_{1} \partial_{2} u+\partial_{2} \partial_{\overline{1}} u=\partial_{\overline{1}} \partial_{\overline{2}} u+\partial_{1} \partial_{\overline{2}} u=0
$$

By direct calculation, using (5.2), we see that at $q_{1}$,

$$
\begin{aligned}
P\left(\ln |\nabla u|^{2}\right)|\nabla u|^{2} \frac{\operatorname{det} g^{\prime}}{\operatorname{det} g} & \\
= & 2 g^{\prime i \bar{j}}\left\{\frac{\partial^{2}|\nabla u|^{2}}{\partial z_{i} \partial \bar{z}_{j}}-\frac{\partial|\nabla u|^{2}}{\partial z_{i}} \frac{\partial|\nabla u|^{2}}{\partial \bar{z}_{j}} \cdot \frac{1}{|\nabla u|^{2}}\right\} \frac{\operatorname{det} g^{\prime}}{\operatorname{det} g} \\
= & 4 g^{\prime i \bar{j}}\left(\partial_{i} \partial_{\bar{j}} \partial_{p} u \partial_{\bar{p}} u+\partial_{i} \partial_{\bar{j}} \partial_{\bar{p}} u \partial_{p} u\right) \frac{\operatorname{det} g^{\prime}}{\operatorname{det} g} \\
& -4 g^{\prime i \bar{j}}\left(\partial_{i} \partial_{\overline{1}} u \partial_{\overline{1}} \partial_{\bar{j}} u+\partial_{i} \partial_{1} u \partial_{1} \partial_{\bar{j}} u\right) \frac{\operatorname{det} g^{\prime}}{\operatorname{det} g} \\
& +4 g^{\prime i \bar{j}}\left(\partial_{i} \partial_{\overline{2}} u \partial_{2} \partial_{\bar{j}} u\right) \frac{\operatorname{det} g^{\prime}}{\operatorname{det} g}+2 g^{\prime i \bar{j}} R_{i \bar{j}}^{1 \overline{1}}|\nabla u|^{2} \frac{\operatorname{det} g^{\prime}}{\operatorname{det} g} \\
& +4 g^{\prime i \bar{j}}\left(\partial_{i} \partial_{2} u \partial_{\overline{2}} \partial_{\bar{j}} u\right) \frac{\operatorname{det} g^{\prime}}{\operatorname{det} g} .
\end{aligned}
$$


The last term $4 g^{\prime i \bar{j}}\left(\partial_{i} \partial_{2} u \partial_{\overline{2}} \partial_{\bar{j}} u\right) \frac{\operatorname{det} g^{\prime}}{\operatorname{det} g} \geq 0$. So, we only need to estimate the first four terms. By equation and (5.1), the first term in (5.5) is

$$
\begin{aligned}
4 g^{\prime i \bar{j}} & \left(\partial_{i} \partial_{\bar{j}} \partial_{p} u \partial_{\bar{p}} u+\partial_{i} \partial_{\bar{j}} \partial_{\bar{p}} u \partial_{p} u\right) \frac{\operatorname{det} g^{\prime}}{\operatorname{det} g} \\
= & 2\left(e^{u}-f e^{-u}\right) \nabla \triangle u \cdot \nabla u-16\left(\nabla\left(\frac{\operatorname{det} u_{i \bar{j}}}{\operatorname{det} g_{i \bar{j}}}\right) \cdot \nabla u\right) \\
= & 2\left(e^{u}-f e^{-u}\right)\{\nabla \triangle u \cdot \nabla u\}-2\left\{\nabla \triangle\left(e^{u}+f e^{-u}\right) \cdot \nabla u\right\} \\
= & -2\left(e^{u}+f e^{-u}\right) \triangle u|\nabla u|^{2}-2\left(e^{u}-f e^{-u}\right)|\nabla u|^{4} \\
& -4 e^{-u}(\nabla u \cdot \nabla f)|\nabla u|^{2}+2 e^{-u} \triangle f|\nabla u|^{2} \\
& -2\left(e^{u}+f e^{-u}\right)\left(\nabla|\nabla u|^{2} \cdot \nabla u\right)+2 e^{-u} \triangle u(\nabla u \cdot \nabla f) \\
& -2 e^{-u}(\nabla u \cdot \nabla \triangle f)+4 e^{-u}(\nabla(\nabla u \cdot \nabla f) \cdot \nabla u) \\
= & -2\left(e^{u}+f e^{-u}\right) \triangle u|\nabla u|^{2}-2\left(e^{u}-f e^{-u}\right)|\nabla u|^{4} \\
& -4 e^{-u}(\nabla u \cdot \nabla f)|\nabla u|^{2}+2 e^{-u} \triangle f|\nabla u|^{2} \\
& +2 \frac{v^{\prime}(u)}{v(u)}\left(e^{u}+f e^{-u}\right)|\nabla u|^{4}+2 e^{-u} \triangle u(\nabla u \cdot \nabla f) \\
& -2 e^{-u}(\nabla u \cdot \nabla \triangle f)+4 e^{-u}(\nabla(\nabla u \cdot \nabla f) \cdot \nabla u) .
\end{aligned}
$$

But from (5.2), (5.3) and (5.4),

$$
\begin{aligned}
\nabla(\nabla u \cdot \nabla f) \cdot \nabla u= & \left\{-\frac{\sqrt{2}}{4} \frac{v^{\prime}(u)}{v(u)}|\nabla u|\left(\partial_{1} f+\partial_{\overline{1}} f\right)\right. \\
& \left.+\frac{1}{2}\left(\partial_{1} \partial_{1} f+2 \partial_{1} \partial_{\overline{1}} f+\partial_{\overline{1}} \partial_{\overline{1}} f\right)\right\}|\nabla u|^{2} \\
\geq & -\left(C_{3}|\nabla u|+C_{3}\right)|\nabla u|^{2},
\end{aligned}
$$

where in the last inequality $C_{3}$ depends only on $\sup u, \inf u$ and $f$. In the following, $C_{3}$ may mean different constants in the different equations that depend only on $\sup u, \inf u, f$ and $S$. Inserting (5.7) into (5.6) and applying the Schwarz inequality, the first term in (5.5) can be estimated to be

$$
\begin{aligned}
4 g^{\prime i \bar{j}} & \left(\partial_{i} \partial_{\bar{j}} \partial_{p} u \partial_{\bar{p}} u+\partial_{i} \partial_{\bar{j}} \partial_{\bar{p}} u \partial_{p} u\right) \frac{\operatorname{det} g^{\prime}}{\operatorname{det} g} \\
\geq & -2\left(e^{u}-f e^{-u}\right)|\nabla u|^{4}+2 \frac{v^{\prime}(u)}{v(u)}\left(e^{u}+f e^{-u}\right)|\nabla u|^{4} \\
\quad & +2\left(e^{u}+f e^{-u}\right)\left(e^{u}-f e^{-u}-\triangle u\right)|\nabla u|^{2}
\end{aligned}
$$




$$
\begin{aligned}
& -\left(e^{u}-f e^{-u}-\triangle u\right)\left(C_{3}|\nabla u|+C_{3}\right) \\
& -C_{3}|\nabla u|^{3}-C_{3}|\nabla u|^{2}-C_{3}|\nabla u|-C_{3} .
\end{aligned}
$$

Next we compute the second term in (5.5):

$$
\begin{aligned}
- & 4 g^{\prime i \bar{j}}\left(\partial_{i} \partial_{\overline{1}} u \partial_{\overline{1}} \partial_{\bar{j}} u+\partial_{i} \partial_{1} u \partial_{1} \partial_{\bar{j}} u\right) \frac{\operatorname{det} g^{\prime}}{\operatorname{det} g} \\
= & -4\left(e^{u}-f e^{-u}\right)\left(\partial_{i} \partial_{\overline{1}} u \partial_{\overline{1}} \partial_{\bar{i}} u+\partial_{i} \partial_{1} u \partial_{1} \partial_{\bar{i}} u\right) \\
& +2 \times 8 \frac{\operatorname{det} u_{i \bar{j}}}{\operatorname{det} g_{i \bar{j}}}\left(\partial_{\overline{1}} \partial_{\overline{1}} u+\partial_{1} \partial_{1} u\right) \\
= & -4\left(e^{u}-f e^{-u}\right)\left(\partial_{i} \partial_{\overline{1}} u \partial_{\overline{1}} \partial_{\bar{i}} u+\partial_{i} \partial_{1} u \partial_{1} \partial_{\bar{i}} u\right) \\
& +2\left(e^{u}-f e^{-u}\right)(\triangle u)\left(\partial_{\overline{1}} \partial_{\overline{1}} u+\partial_{1} \partial_{1} u\right) \\
& +2\left\{\left(e^{u}+f e^{-u}\right)|\nabla u|^{2}+e^{-u} \triangle f\right. \\
& \left.-2 e^{-u} \nabla u \cdot \nabla f\right\}\left(\partial_{\overline{1}} \partial_{\overline{1}} u+\partial_{1} \partial_{1} u\right) .
\end{aligned}
$$

The first two terms in (5.9) are equal to

$$
\begin{aligned}
& -4\left(e^{u}-f e^{-u}\right)\left(\partial_{i} \partial_{\overline{1}} u \partial_{\overline{1}} \partial_{\bar{i}} u+\partial_{i} \partial_{1} u \partial_{1} \partial_{\bar{i}} u\right) \\
+ & 2\left(e^{u}-f e^{-u}\right)(\triangle u)\left(\partial_{\overline{1}} \partial_{\overline{1}} u+\partial_{1} \partial_{1} u\right) \\
= & 4\left(e^{u}-f e^{-u}\right)\left(\partial_{\overline{1}} \partial_{\overline{1}} u+\partial_{1} \partial_{1} u\right) \partial_{2} \partial_{\overline{2}} u \\
& -4\left(e^{u}-f e^{-u}\right)\left(\partial_{2} \partial_{\overline{1}} u \partial_{\overline{1}} \partial_{\overline{2}} u+\partial_{2} \partial_{1} u \partial_{1} \partial_{\overline{2}} u\right) .
\end{aligned}
$$

From (5.3) we find

$$
\partial_{1} \partial_{1} u+\partial_{\overline{1}} \partial_{\overline{1}} u=-\frac{v^{\prime}(u)}{v(u)}|\nabla u|^{2}-2 \partial_{1} \partial_{\overline{1}} u
$$

Inserting (5.11) and (5.4) into (5.10), and using equation and the Schwarz inequality, we get

$$
\begin{aligned}
- & 4\left(e^{u}-f e^{-u}\right)\left(\partial_{i} \partial_{\overline{1}} u \partial_{\overline{1}} \partial_{\bar{i}} u+\partial_{i} \partial_{1} u \partial_{1} \partial_{\bar{i}} u\right) \\
+ & 2\left(e^{u}-f e^{-u}\right)(\triangle u)\left(\partial_{\overline{1}} \partial_{\overline{1}} u+\partial_{1} \partial_{1} u\right) \\
= & -4\left(e^{u}-f e^{-u}\right) \frac{v^{\prime}(u)}{v(u)} u_{2 \overline{2}}|\nabla u|^{2}-\left(e^{u}-f e^{-u}\right)^{2}(\triangle u) \\
& -\left(e^{u}-f e^{-u}\right)\left\{\left(e^{u}+f e^{-u}\right)|\nabla u|^{2}\right. \\
& \left.+e^{-u} \triangle f-2 e^{-u} \nabla u \cdot \nabla f\right\}
\end{aligned}
$$




$$
\begin{aligned}
\geq & -4\left(e^{u}-f e^{-u}\right) \frac{v^{\prime}(u)}{v(u)} u_{2 \overline{2}}|\nabla u|^{2} \\
& +\left(e^{u}-f e^{-u}\right)^{2}\left(e^{u}-f e^{-u}-\triangle u\right) \\
& -C_{3}|\nabla u|^{2}-C_{3}|\nabla u|-C_{3} \\
\geq & -4\left(e^{u}-f e^{-u}\right) \frac{v^{\prime}(u)}{v(u)} u_{2 \overline{2}}|\nabla u|^{2}-C_{3}|\nabla u|^{2}-C_{3}|\nabla u|-C_{3} .
\end{aligned}
$$

Applying (5.11) and the Schwarz inequality, the third term in (5.9) can be estimated:

$$
\begin{aligned}
& 2\left\{\left(e^{u}+f e^{-u}\right)|\nabla u|^{2}+e^{-u} \triangle f-2 e^{-u} \nabla u \cdot \nabla f\right\}\left(\partial_{\overline{1}} \partial_{\overline{1}} u+\partial_{1} \partial_{1} u\right) \\
& \geq-2\left(e^{u}+f e^{-u}\right) \frac{v^{\prime}(u)}{v(u)}|\nabla u|^{4}-C_{3}|\nabla u|^{3}-C_{3}|\nabla u|^{2} \\
& \quad-4\left\{\left(e^{u}+f e^{-u}\right)|\nabla u|^{2}+e^{-u} \triangle f-2 e^{-u} \nabla u \cdot \nabla f\right\} u_{1 \overline{1}} .
\end{aligned}
$$

Inserting (5.12) and (5.13) into (5.9), we get the following estimate of the second term in (5.5)

$$
\begin{aligned}
- & 4 g^{\prime i \bar{j}}\left(\partial_{i} \partial_{\overline{1}} u \partial_{\overline{1}} \partial_{\bar{j}} u+\partial_{i} \partial_{1} u \partial_{1} \partial_{\bar{j}} u\right) \frac{\operatorname{det} g^{\prime}}{\operatorname{det} g} \\
\geq & -2\left(e^{u}+f e^{-u}\right) \frac{v^{\prime}(u)}{v(u)}|\nabla u|^{4}-4\left(e^{u}-f e^{-u}\right) \frac{v^{\prime}(u)}{v(u)} u_{2 \overline{2}}|\nabla u|^{2} \\
& -4\left\{\left(e^{u}+f e^{-u}\right)|\nabla u|^{2}+e^{-u} \triangle f-2 e^{-u} \nabla u \cdot \nabla f\right\} u_{1 \overline{1}} \\
& -C_{3}|\nabla u|^{3}-C_{3}|\nabla u|^{2}-C_{3}|\nabla u|-C_{3} .
\end{aligned}
$$

The third term in (5.5) is

$$
\begin{aligned}
& 4 g^{\prime i \bar{j}}\left(\partial_{i} \partial_{\overline{2}} u \partial_{2} \partial_{\bar{j}} u\right) \cdot \frac{\operatorname{det} g^{\prime}}{\operatorname{det} g} \\
&=-4\left(e^{u}-f e^{-u}\right) \operatorname{det}\left(u_{i \bar{j}}\right)+2\left(e^{u}-f e^{-u}\right)(\triangle u) u_{2 \overline{2}} \\
& \quad-2 \triangle\left(e^{u}+f e^{-u}\right) \cdot u_{2 \overline{2}} \\
&=-4\left(e^{u}-f e^{-u}\right) \operatorname{det}\left(u_{i \bar{j}}\right) \\
& \quad-2\left\{\left(e^{u}+f e^{-u}\right)|\nabla u|^{2}+e^{-u} \triangle f-2 e^{-u} \nabla u \cdot \nabla f\right\} u_{2 \overline{2}} \\
& \geq \frac{1}{2}\left(e^{u}-f e^{-u}\right)^{2}\left(e^{u}-f e^{-u}-\triangle u\right)-C_{3}|\nabla u|-C_{3}|\nabla u|^{2} \\
&-2\left\{\left(e^{u}+f e^{-u}\right)|\nabla u|^{2}+e^{-u} \triangle f-2 e^{-u} \nabla u \cdot \nabla f\right\} u_{2 \overline{2}} \\
& \geq-2\left\{\left(e^{u}+f e^{-u}\right)|\nabla u|^{2}+e^{-u} \triangle f-2 e^{-u} \nabla u \cdot \nabla f\right\} u_{2 \overline{2}} \\
&-C_{3}|\nabla u|-C_{3}|\nabla u|^{2} .
\end{aligned}
$$


Combining the following two terms in (5.14) and (5.15),

$$
\begin{aligned}
- & 4\left\{\left(e^{u}+f e^{-u}\right)|\nabla u|^{2}+e^{-u} \triangle f-2 e^{-u} \nabla u \cdot \nabla f\right\} u_{1 \overline{1}} \\
- & 2\left\{\left(e^{u}+f e^{-u}\right)|\nabla u|^{2}+e^{-u} \triangle f-2 e^{-u} \nabla u \cdot \nabla f\right\} u_{2} \overline{2} \\
\geq & \frac{1}{2}\left\{\left(e^{u}+f e^{-u}\right)|\nabla u|^{2}+e^{-u} \triangle f-2 e^{-u} \nabla u \cdot \nabla f\right\}\left(e^{u}-f e^{-u}-4 u_{1 \overline{1}}\right) \\
& +\left(e^{u}+f e^{-u}\right)\left(e^{u}-f e^{-u}-\triangle u\right)-\left(e^{u}-f e^{-u}-\triangle u\right)\left(C_{3}|\nabla u|+C_{3}\right) \\
& -C_{3}|\nabla u|^{3}-C_{3}|\nabla u|^{2}-C_{3}|\nabla u|-C_{3} \\
\geq & \left(e^{u}+f e^{-u}\right)\left(e^{u}-f e^{-u}-\triangle u\right)-\left(e^{u}-f e^{-u}-\triangle u\right)\left(C_{3}|\nabla u|+C_{3}\right) \\
& -C_{3}|\nabla u|^{3}-C_{3}|\nabla u|^{2}-C_{3}|\nabla u|-C_{3},
\end{aligned}
$$

where in the last inequality we have assumed that

$$
\left(e^{u}+f e^{-u}\right)|\nabla u|^{2}+e^{-u} \triangle f-2 e^{-u} \nabla u \cdot \nabla f \geq 0 .
$$

Otherwise, we could have obtained the estimate of $|\nabla u|^{2}$ at point $q_{1}$. Then, combining (5.14) and (5.15), we get

$$
\begin{aligned}
- & 4 g^{\prime i \bar{j}}\left(\partial_{i} \partial_{\overline{1}} u \partial_{\overline{1}} \partial_{\bar{j}} u+\partial_{i} \partial_{1} u \partial_{1} \partial_{\bar{j}} u-\partial_{i} \partial_{\overline{2}} u \partial_{2} \partial_{\bar{j}} u\right) \frac{\operatorname{det} g^{\prime}}{\operatorname{det} g} \\
\geq & -2\left(e^{u}+f e^{-u}\right) \frac{v^{\prime}(u)}{v(u)}|\nabla u|^{4}-4\left(e^{u}-f e^{-u}\right) \frac{v^{\prime}(u)}{v(u)} u_{2 \overline{2}}|\nabla u|^{2} \\
& -C_{3}|\nabla u|^{3}-C_{3}|\nabla u|^{2}-C_{3}|\nabla u|-C_{3} .
\end{aligned}
$$

Let $R=\operatorname{Supp}\left|R_{i \bar{j}}^{1 \overline{1}}\right|$. The fourth term is

$$
\begin{aligned}
2 g^{\prime i \bar{j}} R_{i \bar{j}}^{1 \overline{1}}|\nabla u|^{2} \frac{\operatorname{det} g^{\prime}}{\operatorname{det} g} & =-8 u^{i \bar{j}} R_{i \bar{j}}^{1 \overline{1}}|\nabla u|^{2} \\
& \geq-8 R \sum_{i, j=1}\left|u_{i \bar{j}}\right||\nabla u|^{2} \\
& \geq-8 R\left((\triangle u)^{2}-8 \operatorname{det} u_{i \bar{j}}\right)^{\frac{1}{2}}|\nabla u|^{2} \\
& \geq-16 R\left(e^{u}-f e^{-u}-\triangle u\right)|\nabla u|^{2}-C_{3}|\nabla u|^{2} .
\end{aligned}
$$


Inserting (5.8), (5.16) and (5.17) into (5.5), we see that

$$
\begin{aligned}
& P\left(\ln |\nabla u|^{2}\right)|\nabla u|^{2} \frac{\operatorname{det} g^{\prime}}{\operatorname{det} g} \\
& \geq\left\{\left(3\left(e^{u}+f e^{-u}\right)-16 R\right)|\nabla u|^{2}\right. \\
& \left.\quad-C_{3}|\nabla u|-C_{3}\right\}\left(e^{u}-f e^{-u}-\triangle u\right) \\
& \quad+\frac{v^{\prime}(u)}{v(u)}\left(e^{u}-f e^{-u}\right)\left(e^{u}-f e^{-u}-4 u_{2 \overline{2}}\right)|\nabla u|^{2} \\
& \quad-2\left(e^{u}-f e^{-u}\right)|\nabla u|^{4}-C_{3}|\nabla u|^{3} \\
& \quad-C_{3}|\nabla u|^{2}-C_{3}|\nabla u|-C_{3} .
\end{aligned}
$$

Next, we compute

$$
\begin{aligned}
P(\ln v) & \frac{\operatorname{det} g^{\prime}}{\operatorname{det} g} \\
= & 2 g^{\prime i \bar{j}}\left(\frac{v^{\prime}}{v} \frac{\partial^{2} u}{\partial z_{i} \partial \bar{z}_{j}}+\frac{v v^{\prime \prime}-v^{\prime 2}}{v^{2}} \frac{\partial u}{\partial z_{i}} \frac{\partial u}{\partial \bar{z}_{j}}\right) \frac{\operatorname{det} g^{\prime}}{\operatorname{det} g} \\
= & \frac{v^{\prime}}{v}\left\{\left(e^{u}-f e^{-u}\right) \triangle u-16 \frac{\operatorname{det}\left(u_{i \bar{j}}\right)}{\operatorname{det} g}\right\} \\
& +\frac{v v^{\prime \prime}-v^{\prime 2}}{v^{2}} g^{\prime 1 \overline{1}}|\nabla u|^{2} \frac{\operatorname{det} g^{\prime}}{\operatorname{det} g} \\
= & -\frac{v^{\prime}(u)}{v(u)}\left(e^{u}-f e^{-u}\right) \triangle u \\
& +\frac{v v^{\prime \prime}-v^{\prime 2}}{v^{2}}|\nabla u|^{2}\left(e^{u}-f e^{-u}-4 u_{2 \overline{2}}\right) \\
& -2 \frac{v^{\prime}(u)}{v(u)}\left\{\left(e^{u}+f e^{-u}\right)|\nabla u|^{2}\right. \\
& \left.+e^{-u} \triangle f-2 e^{-u} \nabla u \cdot \nabla f\right\} \\
\geq & -\frac{v^{\prime}(u)}{v(u)}\left(e^{u}-f e^{-u}\right)\left(e^{u}-f e^{-u}-\triangle u\right) \\
& -2 \frac{v^{\prime}(u)}{v(u)}\left(e^{u}+f e^{-u}\right)|\nabla u|^{2} \\
& +\frac{v v^{\prime \prime}-v^{\prime 2}}{v^{2}}|\nabla u|^{2}\left(e^{u}-f e^{-u}-4 u_{2 \overline{2}}\right)-C_{3}|\nabla u|-C_{3} .
\end{aligned}
$$


From (5.18) and (5.19), we get

$$
\begin{aligned}
P(\ln \mid & \left.\left.\nabla u\right|^{2}+\ln v\right)|\nabla u|^{2} \frac{\operatorname{det} g^{\prime}}{\operatorname{det} g} \\
= & \left\{\left(2\left(e^{u}+f e^{-u}\right)+\frac{v^{\prime}(u)}{v(u)}\left(e^{u}-f e^{-u}\right)\right\}\right. \\
& \times\left(e^{u}-f e^{-u}-\triangle u\right)|\nabla u|^{2} \\
& +\left\{\left(e^{u}+f e^{-u}-16 R\right)|\nabla u|^{2}-C_{3}|\nabla u|-C_{3}\right\} \\
& \times\left(e^{u}-f e^{-u}-\triangle u\right) \\
& +\left\{\frac{v v^{\prime \prime}-v^{\prime 2}}{v^{2}}|\nabla u|^{2}+\frac{v^{\prime}(u)}{v(u)}\left(e^{u}-f e^{-u}\right)\right\} \\
& \times\left(e^{u}-f e^{-u}-4 u_{2}\right)|\nabla u|^{2} \\
& -2\left\{\left(e^{u}-f e^{-u}\right)+\frac{v^{\prime}(u)}{v(u)}\left(e^{u}+f e^{-u}\right)\right\}|\nabla u|^{4} \\
& -C_{3}|\nabla u|^{3}-C_{3}|\nabla u|^{2}-C_{3}|\nabla u|-C_{3} .
\end{aligned}
$$

Take

$$
v(u)=e^{4 \sup u-2 u}+e^{2 u-4 \sup u}>0 .
$$

Then,

$$
\begin{aligned}
v^{\prime}(u) & =-2 e^{4 \sup u-2 u}+2 e^{2 u-4 \sup u}<0, \\
v^{\prime \prime}(u) & =4 e^{4 \sup u-2 u}+4 e^{2 u-4 \sup u}=4 v(u)>0 .
\end{aligned}
$$

So the factor of the first term in (5.20) is

$$
2\left(e^{u}+f e^{-u}\right)+\left(e^{u}-f e^{-u}\right) \frac{v^{\prime}(u)}{v(u)}>0 .
$$

The factor of the third term in (5.20) is

$$
\begin{aligned}
\frac{v v^{\prime \prime}-v^{\prime} 2}{v^{2}}|\nabla u|^{2}+\frac{v^{\prime}}{v}\left(e^{u}-f e^{-u}\right) & =\frac{16}{v^{2}}|\nabla u|^{2}-\frac{e^{u} e^{4 \sup u-2 u}}{e^{4 \sup u-2 u}} \\
& >\frac{16}{e^{4 \sup u-2 \inf u+3}|\nabla u|^{2}-e^{\sup u} .}
\end{aligned}
$$

Choose $A$ such that

$$
C_{1}^{-1} A^{-\kappa / 2}>7^{1 / 3}
$$


then $e^{\inf u}>\frac{1}{3} \ln 7$ and the coefficient of the fourth term in (5.20) is

$$
\begin{aligned}
-2\left\{\left(e^{u}-f e^{-u}\right)+\frac{v^{\prime}}{v}\left(e^{u}+f e^{-u}\right)\right\} \geq & 2 \frac{e^{4 \sup u-u}-3 e^{3 u-u \sup u}}{e^{4 \sup u-2 u}+e^{2 u-4 \sup u}} \\
& \geq \frac{2 e^{4 \sup u-u}-6}{e^{4 \sup u-u}+1}>1 .
\end{aligned}
$$

Choose $A$ such that

$$
C_{1}^{-1} A^{-\kappa / 2}>16 R+1
$$

then $e^{\inf u}>16 R+1$. Applying all above inequalities, we can see that at $q_{1}$

$$
\begin{aligned}
& 0 \geq P\left(\ln |\nabla u|^{2}+\ln v\right)|\nabla u|^{2} \frac{\operatorname{det} g^{\prime}}{\operatorname{det} g} \\
& \geq\left(|\nabla u|^{2}-C_{3}|\nabla u|-C_{3}\right)\left(e^{u}-f e^{-u}-\triangle u\right) \\
& +\left\{\frac{16}{e^{4 \sup u-2 \inf u}+1}|\nabla u|^{2}-e^{\sup u}\right\}\left(e^{u}-f e^{-u}-4 u_{2 \overline{2}}\right)|\nabla u|^{2} \\
& +|\nabla u|^{4}-C_{3}|\nabla u|^{3}-C_{3}|\nabla u|^{2}-C_{3}|\nabla u|-C_{3} \text {. }
\end{aligned}
$$

From above inequality, we can easily see that there is a constant $C_{4}$ depending only on $f, S, \sup u$ and $\inf u$ such that $|\nabla u|^{2}\left(q_{1}\right) \leq C_{4}$.

We have proved that if the function $\ln |\nabla u|^{2}+\ln \left(e^{4 \sup u-2 u}+e^{2 u-\sup u}\right)$ achieves its maximum at $q_{1}$, we get the bound of $|\nabla u|^{2}$ :

$$
\begin{aligned}
|\nabla u|^{2} & \leq C_{4} \frac{\left(e^{4 \sup u-2 u(q)}+e^{2 u(q)-4 \sup u}\right)}{\left(e^{4 \sup u-2 u}+e^{2 u-4 \sup u}\right)} \\
& \leq C_{4} \frac{\left(e^{4 \sup u-2 \inf u}+e^{2 \inf u-4 \sup u}\right)}{\left(e^{2 \sup u}+e^{-2 \sup u}\right)} .
\end{aligned}
$$

\section{Second-order estimate}

We now give the second-order a priori estimate of $u$. We rewrite the equation as

$$
\frac{\operatorname{det} g_{i \bar{j}}^{\prime}}{\operatorname{det} g_{i \bar{j}}}=F,
$$


where

$$
\begin{aligned}
F= & \left(e^{u}-f e^{-u}\right)^{2}+2\left\{\left(e^{u}+f e^{-u}\right)^{2}|\nabla u|^{2}\right. \\
& \left.+e^{-u} \triangle f-2 e^{-u} \nabla u \cdot \nabla f\right\} .
\end{aligned}
$$

Then we can see that if we suppose $A$ is small, $F$ has a positive lower bound. Hence, $e^{u}-f e^{-u}-\triangle u \geq F^{1 / 2}$ also has a positive lower bound. So, to get a second-order estimate of $u$, it sufficient to have an upper estimate of $e^{u}-f e^{-u}-\triangle u$. We fix a point $q_{2}$ and choose normal coordinate at that point for $g_{i \bar{j}}$.

Differentiating (6.1), we have

$$
g^{i \bar{j}} \frac{\partial g_{i \bar{j}}^{\prime}}{\partial z_{k}}=g^{i \bar{j}} \frac{\partial g_{i \bar{j}}}{\partial z_{k}}+\frac{1}{F} \frac{\partial F}{\partial z_{k}}
$$

By differentiating (6.3) again, we obtain

$$
\begin{aligned}
& -g^{\prime i \bar{q}} g^{\prime p \bar{j}} \frac{\partial g_{p \bar{q}}^{\prime}}{\partial \bar{z}_{l}} \frac{\partial g_{i \bar{j}}^{\prime}}{\partial z_{k}}+g^{\prime i \bar{j}} \frac{\partial^{2} g_{i \bar{j}}^{\prime}}{\partial z_{k} \partial \bar{z}_{l}} \\
& =-g^{i \bar{q}} g^{p \bar{j}} \frac{\partial g_{p \bar{q}}}{\partial \bar{z}_{l}} \frac{\partial g_{i \bar{j}}}{\partial z_{k}}+g^{i \bar{j}} \frac{\partial^{2} g_{i \bar{j}}}{\partial z_{k} \partial \bar{z}_{l}}+\frac{1}{F} \frac{\partial^{2} F}{\partial z_{k} \partial \bar{z}_{l}}-\frac{1}{F^{2}} \frac{\partial F}{\partial z_{k}} \frac{\partial F}{\partial \bar{z}_{l}}
\end{aligned}
$$

or

$$
\begin{aligned}
-4 g^{\prime i \bar{j}} \frac{\partial^{4} u}{\partial z_{i} \partial \bar{z}_{j} \partial z_{k} \partial \bar{z}_{l}}= & g^{\prime i \bar{q}} g^{\prime p \bar{j}} \frac{\partial g_{p \bar{q}}^{\prime}}{\partial \bar{z}_{l}} \frac{\partial g_{i \bar{j}}^{\prime}}{\partial z_{k}}-g^{\prime i \bar{j}} \frac{\partial^{2}\left(\left(e^{u}-f e^{-u}\right) g_{i \bar{j}}\right)}{\partial z_{k} \partial \bar{z}_{l}} \\
& -g^{i \bar{q}} g^{p \bar{j}} \frac{\partial g_{p \bar{q}}}{\partial \bar{z}_{l}} \frac{\partial g_{i \bar{j}}}{\partial z_{k}}+g^{i \bar{j}} \frac{\partial^{2} g_{i \bar{j}}}{\partial z_{k} \partial \bar{z}_{l}} \\
& +\frac{1}{F} \frac{\partial^{2} F}{\partial z_{k} \partial \bar{z}_{l}}-\frac{1}{F^{2}} \frac{\partial F}{\partial z_{k}} \frac{\partial F}{\partial \bar{z}_{l}}
\end{aligned}
$$

Contracting (6.4) with $g^{k l}$ and using the fact that the metric $g_{i \bar{j}}$ is Ricci-flat, we find

$$
\begin{aligned}
P(-\triangle u)= & g^{k \bar{l}} g^{\prime i \bar{j}} g^{\prime p \bar{q}} \frac{\partial g_{i \bar{q}}^{\prime}}{\partial z_{k}} \frac{\partial g_{p \bar{j}}^{\prime}}{\partial \bar{z}_{l}}-\frac{1}{2} \triangle\left(e^{u}-f e^{-u}\right) \sum_{i=1}^{2} g^{\prime i \bar{i}} \\
& +\frac{1}{2 F} \triangle F-\frac{1}{F^{2}} g^{k \bar{l}} \frac{\partial F}{\partial z_{k}} \frac{\partial F}{\partial \bar{z}_{l}}+4 g^{\prime i \bar{j}} \frac{\partial^{2} g^{k \bar{l}}}{\partial z_{i} \partial \bar{z}_{j}} \frac{\partial^{2} u}{\partial z_{k} \partial \bar{z}_{l}}
\end{aligned}
$$


Multiplying the above equation by $\frac{\operatorname{det} g_{i \bar{j}}^{\prime}}{\operatorname{det} g_{i \bar{j}}}$ and using (6.3) and (6.1), we see

$$
\begin{aligned}
P(-\triangle u) \frac{\operatorname{det} g_{i \bar{j}}^{\prime}}{\operatorname{det} g_{i \bar{j}}}= & g^{k \bar{l}} g^{\prime i \bar{j}} g^{\prime p \bar{q}}\left(\frac{\partial g_{i \bar{q}}^{\prime}}{\partial z_{k}} \frac{\partial g_{p \bar{j}}^{\prime}}{\partial \bar{z}_{l}}-\frac{\partial g_{i \bar{j}}^{\prime}}{\partial z_{k}} \frac{\partial g_{p \bar{q}}^{\prime}}{\partial \bar{z}_{l}}\right) \frac{\operatorname{det} g_{i \bar{j}}^{\prime}}{\operatorname{det} g_{i \bar{j}}} \\
& +\frac{1}{2} \triangle F-\frac{1}{2} \triangle\left(e^{u}-f e^{-u}\right) \sum_{i=1}^{2} g^{\prime i \bar{i}} \frac{\operatorname{det} g_{i \bar{j}}^{\prime}}{\operatorname{det} g_{i \bar{j}}} \\
& +4 g^{\prime i \bar{j}} \frac{\partial^{2} g^{k \bar{l}}}{\partial z_{i} \partial \bar{z}_{j}} \frac{\partial^{2} u}{\partial z_{k} \partial \bar{z}_{l}} .
\end{aligned}
$$

Now we compute at $q_{2}$

$$
\begin{aligned}
& g^{k \bar{l}} g^{\prime i \bar{j}} g^{\prime p \bar{q}}\left(\frac{\partial g_{i \bar{q}}^{\prime}}{\partial z_{k}} \frac{\partial g_{p \bar{j}}^{\prime}}{\partial \bar{z}_{l}}-\frac{\partial g_{i \bar{j}}^{\prime}}{\partial z_{k}} \frac{\partial g_{p \bar{q}}^{\prime}}{\partial \bar{z}_{l}}\right) \frac{\operatorname{det} g_{i \bar{j}}^{\prime}}{\operatorname{det} g_{i \bar{j}}} \\
&=g^{k \bar{l}}\left(g^{\prime i \bar{j}} g^{\prime p \bar{q}}-g^{\prime i \bar{q}} g^{\prime p \bar{j}}\right) \frac{\partial g_{i \bar{q}}^{\prime}}{\partial z_{k}} \frac{\partial g_{p \bar{j}}^{\prime}}{\partial \bar{z}_{l}} \frac{\operatorname{det} g_{i \bar{j}}^{\prime}}{\operatorname{det} g_{i \bar{j}}} \\
&=g^{k \bar{l}}\left(\frac{\partial g_{2 \overline{1}}^{\prime}}{\partial z_{k}} \frac{\partial g_{1 \overline{2}}^{\prime}}{\partial \bar{z}_{l}}+\frac{\partial g_{1 \overline{2}}^{\prime}}{\partial z_{k}} \frac{\partial g_{2 \overline{1}}^{\prime}}{\partial \bar{z}_{l}}-\frac{\partial g_{1 \overline{1}}^{\prime}}{\partial z_{k}} \frac{\partial g_{2 \overline{2}}^{\prime}}{\partial \bar{z}_{l}}-\frac{\partial g_{2 \overline{2}}^{\prime}}{\partial z_{k}} \frac{\partial g_{1 \overline{1}}^{\prime}}{\partial \bar{z}_{l}}\right) \\
&=16 \sum_{i \neq j}\left(\frac{\partial^{3} u}{\partial z_{i} \partial \bar{z}_{j} \partial z_{k}} \frac{\partial^{3} u}{\partial z_{j} \partial \bar{z}_{i} \partial \bar{z}_{k}}-\frac{\partial^{3} u}{\partial z_{i} \partial \bar{z}_{i} \partial z_{k}} \frac{\partial^{3} u}{\partial z_{j} \partial \bar{z}_{j} \partial \bar{z}_{k}}\right) \\
&+4 \sum_{i} \frac{\partial^{3} u}{\partial z_{i} \partial \bar{z}_{i} \partial z_{k}} \frac{\partial\left(e^{u}-f e^{-u}\right)}{\partial \bar{z}_{k}}+4 \sum_{i} \frac{\partial^{3} u}{\partial z_{i} \partial \bar{z}_{i} \partial \bar{z}_{k}} \frac{\partial\left(e^{u}-f e^{-u}\right)}{\partial z_{k}} \\
&-2 \frac{\partial\left(e^{u}-f e^{-u}\right)}{\partial \bar{z}_{k}} \frac{\partial\left(e^{u}-f e^{-u}\right)}{\partial z_{k}} \\
&= 16 \sum_{i, j} \frac{\partial^{3} u}{\partial z_{i} \partial \bar{z}_{j} \partial z_{k}} \frac{\partial^{3} u}{\partial z_{j} \partial \bar{z}_{i} \partial \bar{z}_{k}}-16 \sum_{i, j} \frac{\partial^{3} u}{\partial z_{i} \partial \bar{z}_{i} \partial z_{k}} \frac{\partial^{3} u}{\partial z_{j} \partial \bar{z}_{j} \partial \bar{z}_{k}} \\
&+2 \nabla(\triangle u) \cdot \nabla\left(e^{u}-f e^{-u}\right)-\left|\nabla\left(e^{u}-f e^{-u}\right)\right|^{2} \\
& \geq-2|\nabla \triangle u|^{2}+2 \nabla(\triangle u) \cdot \nabla\left(e^{u}-f e^{-u}\right)-C_{5},
\end{aligned}
$$

where $C_{5}$ depends on $f, S$ and $u$ up to first-order derivation. In the following, we will use $C_{5}$ to denote a constant with similar dependence and estimate an upper bound for $e^{u}-f e^{-u}-\triangle u$. Assume that $e^{u}-f e^{-u}-\triangle u$ achieves a maximum at point $q_{2}$. By taking normal coordinate at $q_{2}$ for $g_{i \bar{j}}$, we find

$$
\nabla \triangle u=\nabla\left(e^{u}-f e^{-u}\right) .
$$


Inserting (6.8) into (6.7) and then into (6.6), we obtain

$$
\begin{aligned}
P(-\triangle u) \frac{\operatorname{det} g_{i \bar{j}}^{\prime}}{\operatorname{det} g_{i \bar{j}}} \geq & \frac{1}{2} \triangle F+4 g^{\prime i \bar{j}} \frac{\partial^{2} g^{k \bar{l}}}{\partial z_{i} \partial \bar{z}_{j}} \frac{\partial^{2} u}{\partial z_{k} \partial \bar{z}_{l}} \\
& -\frac{1}{2} \triangle\left(e^{u}-f e^{-u}\right) \sum_{i=1}^{2} g^{\prime i \bar{i}} \frac{\operatorname{det} g_{i \bar{j}}^{\prime}}{\operatorname{det} g_{i \bar{j}}}-C_{5}
\end{aligned}
$$

We shall first deal with the second term in the above inequality. Using the equation, the Schwarz inequality, and the fact that the metric $g_{i \bar{j}}$ is Ricci-flat, we have

$$
\begin{aligned}
4 g^{\prime i \bar{j}} & \frac{\partial^{2} g^{k \bar{l}}}{\partial z_{i} \partial \bar{z}_{j}} \frac{\partial^{2} u}{\partial z_{k} \partial \bar{z}_{l}} \\
= & -16\left\{\frac{\partial^{2} g^{k \bar{l}}}{\partial z_{1} \partial \bar{z}_{1}} \frac{\partial^{2} u}{\partial u_{2} \partial \bar{z}_{2}}+\frac{\partial^{2} g^{k \bar{l}}}{\partial z_{2} \partial \bar{z}_{2}} \frac{\partial^{2} u}{\partial u_{1} \partial \bar{z}_{1}}\right\} \frac{\partial^{2} u}{\partial z_{k} \partial \bar{z}_{l}} \\
& -16\left\{\frac{\partial^{2} g^{k \bar{l}}}{\partial z_{1} \partial \bar{z}_{2}} \frac{\partial^{2} u}{\partial u_{2} \partial \bar{z}_{1}}+\frac{\partial^{2} g^{k \bar{l}}}{\partial z_{2} \partial \bar{z}_{1}} \frac{\partial^{2} u}{\partial u_{1} \partial \bar{z}_{2}}\right\} \frac{\partial^{2} u}{\partial z_{k} \partial \bar{z}_{l}} \\
\geq & -64\left(\max R_{i \bar{j} k \bar{l}}\right) \sum_{i j}\left|u_{i \bar{j}}\right|^{2} \\
= & -16\left(\max R_{i \bar{j} k \bar{l}}\right)(\triangle u)^{2}-16\left(\max R_{i \bar{j} k \bar{l}}\right) \times 8 \frac{\operatorname{det} u_{i \bar{j}}}{\operatorname{det} g_{i \bar{j}}}, \\
= & -16\left(\max R_{i \bar{j} k \bar{l}}\right)\left\{(\triangle u)^{2}-\triangle\left(e^{u}+f e^{-u}\right)\right\}, \\
\geq & -16\left(\max R_{i \bar{j} k \bar{l}}\right)(\triangle u)^{2}-C_{5} \triangle u-C_{5},
\end{aligned}
$$

since

$$
\sum_{i=1}^{2} g^{\prime i \bar{i}} \frac{\operatorname{det} g_{i \bar{j}}^{\prime}}{\operatorname{det} g_{i \bar{j}}}=2\left(e^{u}-f e^{-u}-\triangle u\right) .
$$

We can insert (6.10) and (6.11) into (6.9) and obtain

$$
\begin{aligned}
P(- & \triangle u) \frac{\operatorname{det} g_{i \bar{j}}^{\prime}}{\operatorname{det} g_{i \bar{j}}} \\
\geq & \frac{1}{2} \triangle F-\triangle\left(e^{u}-f e^{-u}\right)\left(e^{u}-f e^{-u}-\triangle u\right) \\
& -16\left(\max R_{i \bar{j} k \bar{l}}\right)(\triangle u)^{2}-C_{5} \triangle u-C_{5}
\end{aligned}
$$




$$
\begin{aligned}
\geq & \frac{1}{2} \triangle F+\left\{\left(e^{u}+f e^{-u}\right)\right. \\
& \left.-16\left(\max R_{i \bar{j} k \bar{l}}\right)\right\}(\triangle u)^{2}-C_{5} \triangle u-C_{5} .
\end{aligned}
$$

By computation,

$$
\begin{aligned}
\triangle F=\triangle & \left(e^{u}-f e^{-u}\right)^{2}+2 \triangle\left(e^{u}+f e^{-u}\right)|\nabla u|^{2} \\
& +2\left(e^{u}+f e^{-u}\right) \triangle\left(|\nabla u|^{2}\right) \\
& +2 \nabla\left(e^{u}+f e^{-u}\right) \cdot \nabla\left(|\nabla u|^{2}\right) \\
& +2 \triangle e^{-u} \triangle f+2 e^{-u} \triangle^{2} f+2 \nabla e^{-u} \cdot \nabla \triangle f \\
& -4 \triangle e^{-u} \nabla u \cdot \nabla f \\
& -4 e^{-u} \triangle(\nabla u \cdot \nabla f)-4 \nabla e^{-u} \cdot \nabla(\nabla u \cdot \nabla f) \\
\geq & +2\left(e^{u}+f e^{-u}\right) \triangle\left(|\nabla u|^{2}\right)+2 \nabla\left(e^{u}+f e^{-u}\right) \cdot \nabla\left(|\nabla u|^{2}\right) \\
& -4 e^{-u} \triangle(\nabla u \cdot \nabla f) \\
& -4 \nabla e^{-u} \cdot \nabla(\nabla u \cdot \nabla f)-C_{5} \triangle u-C_{5} .
\end{aligned}
$$

Let $\Gamma=g^{i \bar{j}} g^{k \bar{l}} u_{, i k} u_{, \bar{j}}$, where $u_{, i k}$ indicates the covariant differentiation with respect to the given metric. As we take the normal coordinate at $q_{2}$, we have $\Gamma=g^{i \bar{j}} g^{k \bar{l}} u_{i k} u_{\bar{j} \bar{l}}$. Using (6.8) and the equation, we have

$$
\begin{aligned}
\triangle & |\nabla u|^{2}=4 g^{k \bar{l}} \frac{\partial^{2}}{\partial z_{k} \partial \bar{z}_{l}}\left\{g_{i \bar{j}} u_{i} u_{\bar{j}}\right\} \\
= & 4 g^{k \bar{k}} g^{i \bar{i}}\left\{\frac{\partial^{3} u}{\partial z_{i} \partial z_{k} \partial \bar{z}_{k}} \frac{\partial u}{\partial \bar{z}_{i}}+\frac{\partial^{3} u}{\partial \bar{z}_{i} \partial z_{k} \partial \bar{z}_{k}} \frac{\partial u}{\partial z_{i}}\right\} \\
& +4 g^{i \bar{i}} g^{k \bar{k}}\left\{\frac{\partial^{2} u}{\partial z_{i} \partial z_{k}} \frac{\partial^{2} u}{\partial \bar{z}_{i} \partial \bar{z}_{k}}+\frac{\partial^{2} u}{\partial z_{i} \partial \bar{z}_{k}} \frac{\partial^{2} u}{\partial \bar{z}_{i} \partial z_{k}}\right\} \\
= & \nabla \Delta \cdot \nabla u+(\triangle u)^{2}-8 \frac{\operatorname{det} u_{i \bar{j}}}{\operatorname{det} g_{i \bar{j}}}+4 g^{i \bar{j}} g^{k \bar{l}} u_{i k} u_{\bar{j} \bar{l}} \\
= & \nabla\left(e^{u}-f e^{-u}\right) \cdot \nabla u+(\triangle u)^{2}-\triangle\left(e^{u}+f e^{-u}\right)+4 g^{i \bar{j}} g^{k \bar{l}} u_{i k} u_{\bar{j} \bar{l}} \\
\geq & (\triangle u)^{2}+4 \Gamma-C_{5} \triangle u-C_{5} .
\end{aligned}
$$

Using (6.8) and the Schwarz inequality, we also have

$$
4 e^{-u} \triangle(\nabla u \cdot \nabla f) \geq-C_{5} \sum_{i j}\left|u_{i \bar{j}}\right|-C_{5} \Gamma^{1 / 2}-C_{5}
$$


and

$$
\begin{aligned}
& 2 \nabla\left(e^{u}+f e^{-u}\right) \cdot \nabla\left(|\nabla u|^{2}\right)-4 \nabla e^{-u} \cdot \nabla(\nabla u \cdot \nabla f) \\
& \quad \geq-C_{5} \sum_{i j}\left|u_{i \bar{j}}\right|-C_{5} \Gamma^{\frac{1}{2}}-C_{5} .
\end{aligned}
$$

Then inserting (6.14), (6.15) and (6.16) into (6.13), we see

$$
\begin{aligned}
\triangle F \geq & 2\left(e^{u}+f e^{-u}\right)(\triangle u)^{2}+8\left(e^{u}+f e^{-u}\right) \Gamma-C_{5} \triangle u \\
& -C_{5} \sum_{i j}\left|u_{i \bar{j}}\right|-C_{5} \Gamma^{\frac{1}{2}}-C_{5} \\
\geq & 2\left(e^{u}+f e^{-u}\right)(\triangle u)^{2}-C_{5} \triangle u-C_{5} \sum_{i j}\left|u_{i \bar{j}}\right|-C_{5} .
\end{aligned}
$$

Inserting (6.17) into (6.12), we obtain

$$
\begin{aligned}
P(-\triangle u) \frac{\operatorname{det} g_{i \bar{j}}^{\prime}}{\operatorname{det} g_{i \bar{j}}} \geq & \left\{2\left(e^{u}+f e^{-u}\right)-16\left(\max R_{i \bar{j} k \bar{l}}\right)\right\}(\triangle u)^{2} \\
& -C_{5} \triangle u-C_{5} \sum_{i j}\left|u_{i \bar{j}}\right|-C_{5} .
\end{aligned}
$$

Next, we compute

$$
\begin{aligned}
P\left(e^{u}-f e^{-u}\right) \frac{\operatorname{det} g_{i \bar{j}}^{\prime}}{\operatorname{det} g_{i \bar{j}}} & 2 g^{\prime k \bar{l}} \frac{\partial^{2}\left(e^{u}-f e^{-u}\right)}{\partial z_{k} \partial \bar{z}_{l}} \frac{\operatorname{det} g_{i \bar{j}}^{\prime}}{\operatorname{det} g_{i \bar{j}}} \\
= & \triangle\left(e^{u}-f e^{-u}\right)-8 \sum_{i \neq j}\left\{\partial_{i} \partial_{\bar{i}} u \partial_{j} \partial_{\bar{j}}\left(e^{u}-f e^{-u}\right)\right. \\
& \left.-\partial_{i} \partial_{\bar{j}} u \partial_{j} \partial_{\bar{i}}\left(e^{u}-f e^{-u}\right)\right\} \\
= & \triangle\left(e^{u}-f e^{-u}\right)-2\left(e^{u}+f e^{-u}\right) \triangle\left(e^{u}-f e^{-u}\right) \\
& -8\left(e^{-u}-f e^{-u}\right) \sum_{i \neq j}\left\{\partial_{i} \partial_{\bar{i}} u \partial_{j} u \partial_{\bar{j}} u-\partial_{i} \partial_{\bar{j}} u \partial_{j} u \partial_{\bar{i}} u\right\} \\
& -8 e^{-u} \sum_{i \neq j}\left\{\partial_{i} \partial_{\bar{i}} u\left(\partial_{j} u \partial_{\bar{j}} f+\partial_{\bar{j}} u \partial_{j} f\right)\right.
\end{aligned}
$$




$$
\begin{aligned}
& \left.-\partial_{i} \partial_{\bar{j}} u\left(\partial_{j} u \partial_{\bar{i}} f+\partial_{\bar{i}} u \partial_{j} f\right)\right\} \\
& +8 e^{-u} \sum_{i \neq j}\left\{\partial_{i} \partial_{\bar{i}} u \partial_{j} \partial_{\bar{j}} f-\partial_{i} \partial_{\bar{j}} u \partial_{j} \partial_{\bar{i}} f\right\} \\
\geq & -C_{5} \triangle u-C_{5} \sum_{i j}\left|u_{i \bar{j}}\right|-C_{5} .
\end{aligned}
$$

Combining (6.18) and (6.19), we obtain

$$
\begin{aligned}
& P\left(e^{u}-f e^{-u}-\triangle u\right) \frac{\operatorname{det} g_{i \bar{j}}^{\prime}}{\operatorname{det} g_{i \bar{j}}} \\
& \geq\left\{2\left(e^{u}+f e^{-u}\right)-16\left(\max R_{i \bar{j} k \bar{l}}\right)\right\}(\triangle u)^{2} \\
&-C_{5} \triangle u-C_{5} \sum_{i j}\left|u_{i \bar{j}}\right|-C_{5} \\
& \geq(\triangle u)^{2}-C_{5} \triangle u-C_{5},
\end{aligned}
$$

where we have chosen $A$ such that $A$ satisfies (5.25), from which we find $e^{\inf u}>16 R+1$. Since we assume $e^{u}-f e^{-u}-\triangle u$ achieves the maximum at $q_{2},(6.20)$ implies

$$
(\triangle u)^{2}-C_{5} \triangle u-C \leq 0
$$

from which we obtain an upper bound estimate of $e^{u}-f e^{-u}-\triangle u$.

\section{Estimates for general case}

Multiplying the elliptic condition $e^{u} \omega_{S}+\sqrt{-1} e^{-u} \operatorname{tr}\left(\bar{\partial} B \wedge \partial B^{*} \cdot g_{S}^{-1}\right)$ $-2 \sqrt{-1} \partial \bar{\partial} u>0$ by $p e^{-p u}$ and integrating, we get (4.4):

$$
\begin{aligned}
\int\left|\nabla e^{-p / 2 u}\right|^{2} \frac{\omega_{S}^{2}}{2 !}< & \frac{p}{4} \int e^{-(p-1) u} \frac{\omega_{S}^{2}}{2 !} \\
& +\frac{p}{4} \int e^{-(p+1) u} \frac{\sqrt{-1}}{2} \operatorname{tr}\left(\bar{\partial} B \wedge \partial B^{*} \cdot g^{-1}\right) \wedge \omega_{S} \\
\leq & \frac{p}{4} \int e^{-(p-1) u} \frac{\omega_{S}^{2}}{2 !} .
\end{aligned}
$$

Note that we use $-\sqrt{-1} \operatorname{tr}\left(\bar{\partial} B \wedge \partial B^{*} \cdot g^{-1}\right) \wedge \omega_{S} \geq 0$. We can then follow the discussion in Section 3 to give the estimate $\inf u \geq-\ln C_{1}-\frac{\kappa}{2} \ln A$. If $A$ is small, we can make $\inf u>0$ large. So the term $e^{u}$ always controls the term such as $e^{-u}\left|\operatorname{tr}\left(\bar{\partial} B \wedge \partial B^{*} \cdot g^{-1}\right)\right|$ and all the other estimates can be derived as in the special case. 


\section{Solution for irreducible bundles}

In the last section, we have constructed a solution: $\left(V, \pi^{*} H_{0}, X, \omega_{u}\right)$ for reducible bundles, where $u \in C^{\infty}(S, \mathbb{R})$ solves the equation

$$
\sqrt{-1} \partial \bar{\partial} e^{u} \wedge \omega_{S}+\partial \bar{\partial}\left(e^{-u} \operatorname{tr}\left(\bar{\partial} B \wedge \partial B^{*} \cdot g^{-1}\right)\right)+\partial \bar{\partial} u \wedge \partial \bar{\partial} u=0
$$

and satisfies the elliptic condition

$$
e^{u} \omega_{S}+\sqrt{-1} e^{-u} \operatorname{tr}\left(\bar{\partial} B \wedge \partial B^{*} \cdot g^{-1}\right)-2 \sqrt{-1} \partial \bar{\partial} u>0
$$

and the normalization condition

$$
\int_{S} e^{-u}=A
$$

In this section, we shall obtain a solution for irreducible bundles by perturbing around the above solution $\left(V, \pi^{*} H_{0}, X, \omega_{u}\right)$. We follow the method of Li-Yau [5].

Let $D_{s}^{\prime \prime}$ be a family of holomorphic structures on $E$ over $S, H$ be a Hermitian metric on $E$ over $S$ and $\phi$ be a smooth function on $S$. We want to look for conditions on $\left(D_{s}^{\prime \prime}, H, \phi\right)$ such that under these conditions $\left(V, \pi^{*} D_{s}^{\prime \prime}, \pi^{*} H, X, \omega_{u+\phi}\right)$ is the solution to Strominger's system, where

$$
\omega_{u+\phi}=\pi^{*}\left(e^{u+\phi} \omega\right)+\frac{\sqrt{-1}}{2} \theta \wedge \bar{\theta} .
$$

Fix the metric $H_{0}$ as the reference metric on $E$ over $S$. Then for any Hermitian metric $H$ on $E$, we can define a smooth endomorphism $h$ on $E$ by

$$
\left\langle v_{1}, v_{2}\right\rangle_{H}=<v_{1} \cdot h, v_{2}>_{H_{0}} .
$$

Under this isomorphism, we define $\mathcal{H}(E)_{1}$ be the space of all Hermitian metric on $E$ where the associated endomorphism $h$ has determinant one. Let $\mathcal{C}\left(\omega_{S}\right)=\left\{e^{\phi} \omega_{S}\right\}$ be the space of all Hermitian metrics on $S$, which are conformal to $\omega_{S}$. Let End $^{0} E$ be the vector bundle of traceless Hermitian anti-symmetric endomorphisms of $\left(E, H_{0}\right)$. Let

$$
\mathcal{H}_{0}(S)=\left\{\psi \frac{\omega^{2}}{2 !} \mid \int_{S} \psi \frac{\omega^{2}}{2 !}=0\right\}
$$

We define the operator

$$
\mathbf{L}=\mathbf{L}_{\mathbf{1}} \oplus \mathbf{L}_{\mathbf{2}}: \mathcal{H}_{1}(E) \times \mathcal{C}\left(\omega_{S}\right) \rightarrow \Omega_{\mathbb{R}}^{4}\left(\operatorname{End}^{0} E\right) \oplus \mathcal{H}_{0}(S)
$$


by

$$
\begin{gathered}
\mathbf{L}_{\mathbf{1}}\left(h, e^{\phi} \omega_{S}\right)=e^{\phi} h^{-1 / 2} F_{h} h^{1 / 2} \wedge \omega_{S} \\
\mathbf{L}_{\mathbf{2}}\left(h, e^{\phi} \omega_{S}\right)=\sqrt{-1} \partial \bar{\partial}\left(e^{u+\phi} \omega_{S}\right)+\partial \bar{\partial}\left(e^{-u-\phi} \operatorname{tr}\left(\bar{\partial} A \wedge \partial A^{*} \cdot g_{\omega_{S}}^{-1}\right)\right) \\
+\partial \bar{\partial}(u+\phi) \wedge \partial \bar{\partial}(u+\phi)-\frac{1}{2}\left(\operatorname{tr} F_{h} \wedge F_{h}-\operatorname{tr} F_{H_{0}} \wedge F_{H_{0}}\right) .
\end{gathered}
$$

As $F_{h}=F_{H_{0}}+D_{0}^{\prime \prime}\left(D_{0}^{\prime} h \cdot h^{-1}\right)$ and $F_{H_{0}} \wedge \omega_{S}=0$,

$$
e^{\phi} h^{-1 / 2} F_{h} h^{1 / 2} \wedge \omega_{S}=e^{\phi} D_{0}^{\prime \prime}\left(D_{0}^{\prime} h \cdot h^{-1}\right) \wedge \omega_{S}
$$

and using the notation of paper [9], we get

$$
\operatorname{tr}\left(e^{\phi} h^{-1 / 2} F_{h} h^{1 / 2} \wedge \omega_{S}\right)=e^{\phi} D_{0}^{\prime \prime}\left(D_{0}^{\prime} \operatorname{tr} \log h\right) \wedge \omega_{S}=0
$$

as $\operatorname{det} h=1$. So the image of $\mathbf{L}_{\mathbf{1}}$ lies in $\Omega_{\mathbb{R}}^{4}\left(\right.$ End $\left.^{0} E\right)$. As to $\mathbf{L}_{\mathbf{2}}$, according to $\partial \bar{\partial}$-lemma on K3 surface, the image of $\mathbf{L}_{\mathbf{2}}$ lies in $R\left(d d_{c}\right)$. For any $\sqrt{-1} \partial \bar{\partial} \alpha \in$ $R(\sqrt{-1} \partial \bar{\partial})$, we can write $\sqrt{-1} \partial \bar{\partial} \alpha=\psi \frac{\omega_{S}^{2}}{2 !}$ for some function $\psi$ such that $\int_{S} \psi \frac{\omega^{2}}{2 !}=0$. So the image of $\mathbf{L}_{\mathbf{2}}$ lies in $\mathcal{H}_{0}(S)$. Therefore, the operator $\mathbf{L}$ is well defined.

Proposition 8.1. If $\left(h, e^{\phi} \omega_{S}\right) \in \operatorname{ker} \mathbf{L}$, then $\left(V, \pi^{*} D_{0}^{\prime \prime}, \pi^{*} h, \omega_{u+\phi}\right)$ is a solution to Strominger's system.

Proof. According to [5], $\left(V, \pi^{*} D_{0}^{\prime \prime}, \pi^{*} h, X, \omega_{u+\phi}\right)$ is the solution to Strominger's system if and only if $\left(\pi^{*} h, \omega_{u+\phi}\right)$ lies in the kernel of the operator:

$$
\tilde{\mathbf{L}}=\tilde{\mathbf{L}}_{\mathbf{1}} \oplus \tilde{\mathbf{L}}_{\mathbf{2}} \oplus \tilde{\mathbf{L}}_{\mathbf{3}}: \mathcal{H}(V)_{1} \times \mathcal{H}(X) \rightarrow \Omega_{\mathbb{R}}^{6}\left(\operatorname{End}^{0} V\right) \oplus R\left(d d_{c}\right) \oplus R\left(d_{\omega_{0}}^{*}\right)
$$

defined by

$$
\begin{aligned}
& \tilde{\mathbf{L}}_{\mathbf{1}}(\tilde{h}, \tilde{\omega})=\tilde{h}^{-1 / 2} F_{\tilde{h}} \tilde{h}^{1 / 2} \wedge \tilde{\omega}^{2} \\
& \tilde{\mathbf{L}}_{\mathbf{2}}(\tilde{h}, \tilde{\omega})=\sqrt{-1} \partial \bar{\partial} \tilde{\omega}-\frac{1}{2}\left(\operatorname{tr} F_{\tilde{h}} \wedge F_{\tilde{h}}-\operatorname{tr} R_{\tilde{\omega}} \wedge R_{\tilde{\omega}}\right), \\
& \tilde{\mathbf{L}}_{\mathbf{3}}(\tilde{h}, \tilde{\omega})=*_{\omega_{0}} d\left(\|\Omega\|_{\tilde{\omega}} \tilde{\omega}^{2}\right) .
\end{aligned}
$$


We want to reduce the above operator $\tilde{\mathbf{L}}$ to vector bundle $E$ over $S$. When $(\tilde{h}, \tilde{\omega})=\left(\pi^{*} h, \omega_{u+\phi}\right)$

$$
\begin{aligned}
\tilde{\mathbf{L}}_{\mathbf{1}}\left(\pi^{*} h, \omega_{u+\phi}\right) & =\pi^{*}\left(h^{-1 / 2} F_{h} h^{1 / 2}\right) \wedge\left(\omega_{u+\phi}\right)^{2} \\
& =\pi^{*}\left(h^{-1 / 2} F_{h} h^{1 / 2} \wedge\left(e^{u+\phi} \omega_{S}\right) \wedge\left(\pi^{*}\left(e^{u+\phi} \omega_{S}\right)+\sqrt{-1} \theta \wedge \bar{\theta}\right)\right. \\
& =\pi^{*} e^{u} \cdot \pi^{*} \mathbf{L}_{\mathbf{1}}\left(h, e^{\phi} \omega_{S}\right) \wedge\left(\pi^{*}\left(e^{u+\phi} \omega_{S}\right)+\sqrt{-1} \theta \wedge \bar{\theta}\right),
\end{aligned}
$$

then $\left(h, e^{\phi} \omega_{S}\right) \in \operatorname{ker} \mathbf{L}_{1}$ if and only if $\left(\pi^{*} h, \omega_{u+\phi}\right) \in \operatorname{ker} \tilde{\mathbf{L}}_{1}$.

Next, we consider $\tilde{\mathbf{L}}_{\mathbf{2}}$. When $(\tilde{h}, \tilde{\omega})=\left(\pi^{*} h, \omega_{u+\phi}\right)$, by Proposition 2.3, we have

$$
\begin{aligned}
\operatorname{tr} R_{\omega_{u+\phi}} \wedge R_{\omega_{u+\phi}}= & \pi^{*}\left(\operatorname{tr} R_{e^{(u+\phi)} \omega_{S}} \wedge R_{e^{(u+\phi)} \omega_{S}}\right. \\
& \left.+2 \partial \bar{\partial}\left(e^{-u-\phi} \operatorname{tr}\left(\bar{\partial} A \wedge \partial A^{*} \cdot g_{\omega_{S}}^{-1}\right)\right)\right)
\end{aligned}
$$

Since $\operatorname{tr} R_{\omega_{S}}=0$ and

$$
R_{e^{(u+\phi)} \omega_{S}}=\bar{\partial} \partial(u+\phi) \cdot I+R_{\omega_{S}}
$$

we have

$$
\operatorname{tr} R_{e^{(u+\phi)} \omega_{S}} \wedge R_{e^{(u+\phi)} \omega_{S}}=2 \partial \bar{\partial}(u+\phi) \wedge \partial \bar{\partial}(u+\phi)+\operatorname{tr} R_{\omega_{S}} \wedge R_{\omega_{S}} .
$$

Thus,

$$
\begin{aligned}
\operatorname{tr} R_{\omega_{u+\phi}} \wedge R_{\omega_{u+\phi}}= & \pi^{*}\left\{2 \partial \bar{\partial}(u+\phi) \wedge \partial \bar{\partial}(u+\phi)+\operatorname{tr}\left(R_{\omega_{S}} \wedge R_{\omega_{S}}\right)\right. \\
& \left.+2 \partial \bar{\partial}\left(e^{-u-\phi} \operatorname{tr}\left(\bar{\partial} A \wedge \partial A^{*} \cdot g_{\omega_{S}}^{-1}\right)\right)\right\}
\end{aligned}
$$

Using the above equality, we obtain

$$
\begin{aligned}
\tilde{\mathbf{L}}_{\mathbf{2}}\left(\pi^{*} h, \omega^{u}\right)= & \pi^{*}\left\{\sqrt{-1} \partial \bar{\partial}\left(e^{(u+\phi)} \omega_{S}\right)+\partial \bar{\partial}\left(e^{-u-\phi} \operatorname{tr}\left(\bar{\partial} A \wedge \partial A^{*} \cdot g_{\omega_{S}}^{-1}\right)\right)\right. \\
& \left.+\partial \bar{\partial}(u+\phi) \wedge \partial \bar{\partial}(u+\phi)-\frac{1}{2}\left(\operatorname{tr} F_{h} \wedge F_{h}-\operatorname{tr} F_{H_{0}} \wedge F_{H_{0}}\right)\right\} \\
= & \pi^{*}\left(\mathbf{L}_{\mathbf{2}}\left(h, e^{\phi} \omega_{S}\right)\right)
\end{aligned}
$$

So $\tilde{\mathbf{L}}_{\mathbf{2}}\left(\pi^{*} h, e^{u} \omega\right)=0$ if and only if $\mathbf{L}_{\mathbf{2}}(h, \omega)=0$.

As for $\tilde{\mathbf{L}}_{\mathbf{3}}$, by Theorem 2.1, we always have $\tilde{\mathbf{L}}_{\mathbf{3}}\left(\pi^{*} h, \omega_{u+\phi}\right)=*_{0} d$ $\left(\|\Omega\|_{\omega_{u+\phi}} \omega_{u+\phi}^{2}\right)=0$. 
Since $\left(V, \pi^{*} D_{0}, \pi^{*} H_{0}, \omega_{u}\right)$ is a solution, $\left(I, \omega_{S}\right) \in \operatorname{ker} \mathbf{L}$. Now we follow [5]. Let $\mathbb{R}^{+2}=\left\{T=\left(T_{1}, T_{2}\right) \in \mathbb{R}^{2} \mid T_{i}>0\right\}$ and let $I_{1}, I_{2}$ and $I_{3}$ be the identity endomorphisms of $L_{1}, L_{2}$ and $T^{\prime} S$, respectively. Then the assignment

$$
T=\left(T_{1}, T_{2}\right) \in \mathbb{R}^{+2} \longmapsto h_{T}=T_{1} I_{1} \oplus T_{2} I_{2} \oplus T_{1}^{-1 / 2} T_{2}^{-1 / 2} I_{3}
$$

assigns each $T \in \mathbb{R}^{+2}$ to a Hermitian endomorphism of $E$. Obviously, $F_{h_{T}}=$ $F_{H_{0}}$ and we still have $\left(h_{T}, \omega_{S}\right) \in \operatorname{ker} \mathbf{L}$.

Pick an integer $k$ and a large $p$. We endow the domain and the target of $\mathbf{L}_{\mathbf{1}} \oplus \mathbf{L}_{\mathbf{2}}$ with Banach space structures as follows:

$$
\mathcal{H}_{1}(E)_{L_{k}^{p}} \times \mathcal{C}\left(\omega_{S}\right)_{L_{k}^{p}} \rightarrow \Omega_{\mathbb{R}}^{4}\left(\operatorname{End}^{0} E\right)_{L_{k-2}^{p}} \oplus \mathcal{H}_{0}(S)_{L_{k-2}^{p}} .
$$

Then, $\mathbf{L}_{\mathbf{1}} \oplus \mathbf{L}_{\mathbf{2}}$ becomes a smooth operator, and its linearized operator $\delta \mathbf{L}_{\mathbf{1}} \oplus \delta \mathbf{L}_{\mathbf{2}}$ at a solution $\left(h_{T}, \omega_{S}\right)$ becomes a linear map

$$
\Omega^{0}\left(\operatorname{Her}^{0} E\right)_{L_{k}^{p}} \oplus\left\{\phi \omega_{S}\right\}_{L_{k}^{p}} \rightarrow \Omega_{\mathbb{R}}^{4}\left(\operatorname{End}^{0} E\right)_{L_{k-2}^{p}} \oplus \mathcal{H}_{0}(S)_{L_{k-2}^{p}} .
$$

Here, we use $\operatorname{Her}^{0} E$ to denote the $\mathbb{R}$-subvector bundle of End $E$ consisting of traceless pointwise $<$, >-Hermitian symmetric endomorphisms of $E$ and the canonical isomorphisms $T_{h_{T}} \mathcal{H}_{1}(E)_{L_{k}^{p}} \cong \Omega^{0}\left(\operatorname{Her}^{0} E\right)_{L_{k}^{p}}$. Clearly, we have $T_{\omega_{S}} \mathcal{C}\left(\omega_{S}\right)_{L_{k}^{p}} \cong\left\{\phi \omega_{S}\right\}_{L_{k}^{p}}$.

To study the kernel and the cokernel of $\delta \mathbf{L}_{\mathbf{1}} \oplus \delta \mathbf{L}_{\mathbf{2}}$ at a trivial solution $\left(h_{T}, \omega_{S}\right)$ we need to look at the linear map

$$
F(\delta h)=D_{0}^{\prime \prime} D_{0, h_{T}}^{\prime}(\delta h) \wedge \omega_{S}: \Omega^{0}\left(\operatorname{Her}^{0} E\right)_{L_{k}^{p}} \rightarrow \Omega_{\mathbb{R}}^{4}\left(\operatorname{End}^{0} E\right)_{L_{k-2}^{p}} .
$$

Here, according to our convention, $D_{h_{T}}=D_{0 . h_{T}}^{\prime} \oplus D_{0}^{\prime \prime}$ is the Hermitian connection of $\left(E, D_{0}^{\prime \prime}, h_{T}\right)$ for a $T=\left(T_{1}, T_{2}\right) \in \mathbb{R}^{+2}$. Since $\left(E, D_{0}^{\prime \prime}\right)=L_{1} \oplus L_{2} \oplus$ $T S$ and $\operatorname{deg} L_{i}=0$, the above is a linear elliptic operator of index 0 whose kernel is

$$
V_{0}=\left\{M_{1} \cdot I_{1} \oplus M_{2} \cdot I_{2} \oplus-\left(\frac{M_{1}}{2}+\frac{M_{2}}{2}\right) I_{3}\right\}
$$

and whose cokernel is

$$
V_{1}=\omega_{S}^{2} \cdot V_{0} \subset \Omega_{\mathbb{R}}^{4}\left(\operatorname{End}^{0} E\right)_{L_{k-2}^{p}} .
$$

Let $\mathbf{P}$ be the obvious projection

$$
\mathbf{P}: \Omega_{\mathbb{R}}^{4}\left(\operatorname{End}^{0} E\right)_{L_{k-2}^{p}} \rightarrow \frac{\Omega_{\mathbb{R}}^{4}\left(\operatorname{End}^{0} E\right)_{L_{k-2}^{p}}}{V_{1}} .
$$


Proposition 8.2. Let $\left(S, \omega_{S}\right), \Omega_{S}, H_{0}$ and $T=\left(T_{1}, T_{2}\right) \in \mathbb{R}^{+2}$ be as before. Then, the linear operator

$$
\begin{aligned}
& \mathbf{P} \circ \delta \mathbf{L}_{\mathbf{1}}\left(h_{T}, \omega_{S}\right) \oplus \delta \mathbf{L}_{\mathbf{2}}\left(h_{T}, \omega_{S}\right): \\
& \Omega^{0}\left(\operatorname{Her}^{0} E\right)_{L_{k}^{p}} \oplus\left\{\phi \omega_{S}\right\}_{L_{k}^{p}} \rightarrow \frac{\Omega_{\mathbb{R}}^{4}\left(\operatorname{End}^{0} E\right)_{L_{k-2}^{p}}}{V_{1} \oplus \mathcal{H}_{0}(S)_{L_{k-2}^{p}}}
\end{aligned}
$$

is surjective.

Proof. As $F_{h_{T}}=F_{H_{0}}$ and $\delta \omega=\phi \omega_{S}$ for some smooth function $\phi$,

$$
h_{T}^{-1 / 2} F_{h_{T}} h_{T}^{1 / 2} \wedge \delta \omega=0
$$

Hence,

$$
\delta \mathbf{L}_{1}\left(h_{T}, \omega_{S}\right)(\delta h, \delta \omega)=D_{0}^{\prime \prime} D_{h_{T}}^{\prime} \delta h \wedge \omega_{S}
$$

and

$$
\mathbf{P} \circ \delta \mathbf{L}_{\mathbf{1}}\left(h_{T}, \omega_{S}\right): \Omega^{0}(\operatorname{Her} E)_{L_{k}^{p}} \oplus\left\{\phi \omega_{S}\right\}_{L_{k}^{p}} \rightarrow \frac{\Omega_{\mathbb{R}}^{4}\left(\operatorname{End}^{0} E\right)_{L_{k-2}^{p}}}{V_{1}}
$$

is surjective, where

$$
\operatorname{ker} \mathbf{P} \circ \delta \mathbf{L}_{\mathbf{1}}\left(h_{T}, \omega_{S}\right)=V_{0} \oplus\left\{\phi \omega_{S}\right\}_{L_{k}^{p}}
$$

On the other hand, when $\delta \omega=\phi \omega_{S}$,

$$
\begin{aligned}
\delta \mathbf{L}_{\mathbf{2}}\left(h_{T}, \omega_{S}\right)\left(\delta h, \phi \omega_{S}\right)= & \sqrt{-1} \partial \bar{\partial}\left(e^{u} \phi \omega_{S}\right)-\partial \bar{\partial}\left(e^{-u} \phi \operatorname{tr}\left(\bar{\partial} A \wedge \partial A^{*} \cdot g_{S}^{-1}\right)\right) \\
& +2 \partial \bar{\partial} u \wedge \partial \bar{\partial} \phi-\operatorname{tr} \delta F_{h_{T}}(\delta h) \wedge F_{h_{T}} .
\end{aligned}
$$

Because of $\operatorname{tr} \delta F_{h_{T}}(\delta h) \wedge F_{h_{T}} \in \mathcal{H}_{0}(S)$ and (8.6), we only need to prove that $\delta \mathbf{L}_{\mathbf{2}}\left(h_{T}, \omega_{S}\right): 0 \oplus\left\{\phi \omega_{S}\right\}_{L_{k}^{p}} \rightarrow \mathcal{H}_{0}(S)_{L_{k-2}^{p}}$ is surjective. So, we should solve the following equation:

$$
\begin{aligned}
& \sqrt{-1} \partial \bar{\partial}\left(e^{u} \phi \omega_{S}\right)-\partial \bar{\partial}\left(e^{-u} \phi \operatorname{tr}\left(\bar{\partial} A \wedge \partial A^{*} \cdot g_{S}^{-1}\right)\right) \\
& \quad+2 \partial \bar{\partial} u \wedge \partial \bar{\partial} \phi=\psi \frac{\omega_{S}^{2}}{2 !}
\end{aligned}
$$

for any $\psi \in L_{k-2}^{p}$ such that $\int \psi=0$. If we define the linear operator $L$ from $L_{k}^{p}$ to $L_{k-2}^{p}$ by

$$
L(\phi)=\sqrt{-1} \partial \bar{\partial}\left(e^{u} \phi \omega_{S}\right)-\partial \bar{\partial}\left(e^{-u} \phi \operatorname{tr}\left(\bar{\partial} A \wedge \partial A^{*} \cdot g_{S}^{-1}\right)\right)+2 \partial \bar{\partial} u \wedge \partial \bar{\partial} \phi,
$$


then $L$ is the linearized operator of Equation (8.2). We shall prove $\operatorname{ker} L^{*}=$ $\mathbb{R}$ and $\operatorname{dim} \operatorname{ker} L=1$ so that $\mathcal{H}_{0}(S) \perp \operatorname{ker} L^{*}$. From the elliptic condition, the operator $L$ is elliptic and Equation (8.7) has a solution $\phi \in L_{k}^{p}$ for any $\psi \in L_{k-2}^{p}$ such that $\int \psi=0$. Thus we have proven that $P \circ \delta L_{1} \oplus \delta L_{2}$ is surjective and it's kernel is $V_{0} \oplus \operatorname{ker} L$.

Now we deform the holomorphic structure $D_{0}^{\prime \prime}$. The following proposition is due to Jun Li.

Proposition 8.3. There is a family $D_{s}^{\prime \prime}$ of deformations of holomorphic structures of $E$ so that its $k$-th order for $k<m$ Kodaira-Spencer class $\kappa$ all vanish while its $m$-th order Kodaira-Spencer class has non-vanishing summands in $H^{1}\left(L_{i}^{\vee} \otimes T S\right)$ and $H^{1}\left(T S^{\vee} \otimes L_{i}\right)$.

Proof. Since $\operatorname{deg} L_{i}=0$ and $T S$ is slope stable with respect to the Hodge class $\omega_{S}, L_{i}^{\vee} \otimes T S$ has no global sections. Using the Serre duality, $H^{2}\left(L_{i} \otimes\right.$ $T S)=0$ as well. Thus, to compute $H^{1}\left(L_{i}^{\vee} \otimes T S\right)$, we use Riemann-Roch for K3 surfaces

$$
\chi\left(L_{i}^{\vee} \otimes T S\right)=\frac{1}{2} c_{1}\left(L_{i}^{\vee} \otimes T S\right)^{2}+2 \chi\left(\mathcal{O}_{S}\right)-c_{2}\left(L_{i}^{\vee} \otimes T S\right) .
$$

Because $c_{1}\left(L_{i}^{\vee} \otimes T S\right)=-2 c_{1}\left(L_{i}\right)$ and $c_{2}\left(L_{i}^{\vee} \otimes T S\right)=c_{2}(T S)+c_{1}\left(L_{i}\right)^{2}=$ $20+c_{1}\left(L_{i}\right)^{2}$, we have

$$
h^{1}\left(L_{i}^{\vee} \otimes T S\right)=16-c_{1}\left(L_{i}\right)^{2} \geq 16 .
$$

Here, the last inequality follows from $c_{1}\left(L_{i}\right) \cdot \omega_{S}=0$ and the Hodge index theorem.

For the same reason, we have

$$
h^{1}\left(T S^{\vee} \otimes L_{i}\right) \geq 16 .
$$

Now consider the vector bundle

$$
E=L_{1} \oplus L_{2} \oplus T S
$$

The extension group $\operatorname{Ext}^{1}(E, E)$ has summands

$$
\operatorname{Ext}^{1}\left(L_{i}, T S\right) \text { and } \operatorname{Ext}^{1}\left(T S, L_{i}\right)
$$

which are $H^{1}\left(L_{i}^{\vee} \otimes T S\right)$ and $H^{1}\left(T S^{\vee} \otimes L_{i}\right)$, respectively; hence they have positive dimension. Thus, we can find a direction $\eta \in \operatorname{Ext}^{1}(E, E)$ that has non-trivial components in the desired factors (8.8). 
It remains to show that $\eta$ can be realized as the tangent of an actual deformation. But this may not be true since the obstruction to deformation of $E$ is the traceless part of $\operatorname{Ext}^{2}(E, E)$, which is isomorphic to two copies of $H^{2}\left(\mathcal{O}_{S}\right) \cong \mathbb{C}$. What is true is that there is a family of deformations of holomorphic structures of $E$, denoted by $\bar{\partial}_{s}$, so that

$$
\left.\frac{d^{k}}{d s^{k}} \bar{\partial}_{s}\right|_{s=0}=0, \quad k<m
$$

and

$$
\left.\frac{d^{m}}{d s^{m}} \bar{\partial}_{s}\right|_{s=0} \neq 0
$$

has non-trivial exponents in (8.8).

Actually, we can define the Kuranish map $K: \mathcal{U} \rightarrow \operatorname{Ext}^{2}(E, E)$, where $\mathcal{U}$ is some open neighborhood of origin in $\operatorname{Ext}^{1}(E, E) . K$ is the holomorphic map and the complex analytic variety $\mathcal{X}=K^{-1}(0)$ is the parametric space of all holomorphic structures on $E$ near $D_{0}^{\prime \prime}$. Considering the dimensions of $\operatorname{Ext}^{1}(E, E)$ and $\operatorname{Ext}^{2}(E, E)$, we can choose an element

$$
\eta=\left(\begin{array}{ccc}
0 & 0 & C_{13} \\
0 & 0 & C_{23} \\
C_{31} & C_{32} & C_{33}
\end{array}\right) \in \operatorname{Ext}^{1}(\mathcal{E}, \mathcal{E})
$$

such that $C_{i 3} \neq 0$ and $C_{3 j} \neq 0$ for $1 \leq i, j \leq 2$ and $\eta$ belongs to the tangent cone of $\mathcal{X}$ at the point $D_{0}^{\prime \prime}$. So, there is a curve $D_{s}^{\prime \prime}$ of degree $m$ of smooth deformation of the holomorphic structure $D_{0}^{\prime \prime}$. If we write

$$
D_{s}^{\prime \prime}=D_{0}^{\prime \prime}+A_{s}, \quad A_{s} \in \Omega^{0,1}\left(\operatorname{End}^{0} E\right),
$$

then $A_{0}^{(k)}=0$ for $k<m$ and $A_{0}^{(m)}=\eta$. We assume that $C_{i j}$ are $D_{0}^{\prime \prime}$-harmonic. Because Pic $S$ is discrete, we can assume further that $\operatorname{tr} A_{s}=0$ for all $s$.

With the connection forms $A_{s}$, the metric $H_{0}$ and the Kähler form $\omega_{S}$ so chosen, we can now define operators

$$
\mathbf{L}_{\mathbf{s}, \mathbf{1}} \oplus \mathbf{L}_{\mathbf{s}, \mathbf{2}}: \mathcal{H}_{1}(E)_{L_{k}^{p}} \times \mathcal{C}\left(\omega_{S}\right)_{L_{k}^{p}} \rightarrow \Omega_{\mathbb{R}}^{4}\left(\operatorname{End}^{0} E\right)_{L_{k-2}^{p}} \oplus \mathcal{H}_{0}(S)_{L_{k-2}^{p}},
$$

with $\mathbf{L}_{\mathbf{s}, \mathbf{i}}$ defined as in (8.3) and (8.4) of which the curvature form $F_{h}$ is replaced by the Hermitian curvature of $\left(E, D_{s}^{\prime \prime}, h\right)$ :

$$
F_{s, h}=D_{s, h} \circ D_{s, h}
$$


From [5], we have

$$
F_{s, h}=F_{s}+D_{s}^{\prime \prime}\left(D_{s}^{\prime} h \cdot h\right)
$$

and

$$
F_{s}=F_{0}+\left(D_{0}^{\prime \prime}+D_{0}^{\prime}\right)\left(A_{s}-A_{s}^{*}\right)-\left(A_{s}-A_{s}^{*}\right) \wedge\left(A_{s}-A_{S}^{*}\right) .
$$

Because $\operatorname{tr} A_{s}=0$, det $h=1$ and $F_{0} \wedge \omega_{S}=0$,

$$
\operatorname{tr} \mathbf{L}_{\mathbf{s}, \mathbf{1}}\left(h, e^{\phi} \omega_{S}\right)=e^{\phi} \operatorname{tr} h^{-1 / 2} F_{s, h} h^{1 / 2} \wedge \omega_{S}=0 .
$$

So, $\mathbf{L}_{\mathbf{s}, \mathbf{1}}$ still lies in $\Omega_{\mathbb{R}}^{4}\left(\operatorname{End}^{0} E\right)_{L_{k-2}^{p}}$. Let $\mathbf{P}$ be the projection from $\Omega_{\mathbb{R}}^{4}\left(\operatorname{End}^{0} E\right)_{L_{k-2}^{p}} \oplus \mathcal{H}_{0}(S)_{L_{k-2}^{p}}$ to $\Omega_{\mathbb{R}}^{4}\left(\operatorname{End}^{0} E\right)_{L_{k-2}^{p}} / V_{1} \oplus \mathcal{H}_{0}(S)_{L_{k-2}^{p}}$. Because we have proven that the linearized operator of $\mathbf{P} \circ \mathbf{L}_{\mathbf{0 , 1}} \oplus \mathbf{L}_{\mathbf{0 , 2}}$ is surjective at $\left(h_{T_{0}}, \omega_{S}\right)$, the implicity theorem implies that for sufficiently small $s$, there are smooth solutions $\left(h_{s, T}, \omega_{s, T}\right)$ to $\mathbf{P} \circ \mathbf{L}_{\mathbf{s}, \mathbf{1}} \oplus \mathbf{L}_{\mathbf{s}, \mathbf{2}}=0$ near $\left(h_{T_{0}}, \omega_{S}\right)$. We can assume that the solutions $\left(h_{s, T}, \omega_{s, T}\right)$ can be parameterized by

$$
(s, T) \in[0, a) \times B_{\epsilon}\left(T_{0,1}\right) \times B_{\epsilon}\left(T_{0,2}\right),
$$

where $T_{0}=\left(T_{0,1}, T_{0,2}\right)$. For simplicity, we denote by $F_{s, T}$ the curvature of the Hermitian vector bundle $\left(E, D_{s}^{\prime \prime}, h_{s, T}\right)$. By our construction, it satisfies

$$
\mathbf{L}_{\mathbf{s}, \mathbf{1}}\left(H_{s, T}, \omega_{s, T}\right) \equiv 0 \quad \bmod V_{1}, \quad \mathbf{L}_{\mathbf{s}, \mathbf{2}}\left(H_{s, T}, \omega_{s, T}\right)=0
$$

Hence, to find solutions to $\mathbf{L}_{\mathbf{s}, \mathbf{1}} \oplus \mathbf{L}_{\mathbf{s}, \mathbf{2}}=0$, it suffices to investigate the vanishing loci of the functional $\mathbf{r}_{\mathbf{i}}(\mathbf{s}, \cdot)$ from $B_{\epsilon}\left(T_{0, i}\right)$ to the Lie algebra $u\left(L_{i}\right)$ defined by

$$
\mathbf{r}_{i}(s, T)=\int_{X}\left[\mathbf{L}_{s, T}\left(H_{s, T}, \omega_{s, T}\right)\right]_{i} \wedge \omega_{S}
$$

where $[\cdot]_{i}$ is the projection from $\Omega_{\mathbb{R}}^{\bullet}\left(\operatorname{End}^{0} E\right)$ to $\Omega_{\mathbb{R}}^{\bullet}\left(u\left(L_{i}\right)\right)$ and $u\left(L_{i}\right)$ is the bundle of $<,>$-Hermitian anti-symmetric endomorphisms of $L_{i}$.

We shall compute $\mathbf{r}_{i}^{(k)}(0, T)$ for all $T$ and for all $k \leq 2 m$. Because $\omega_{s, T} \in$ $\mathcal{C}\left(\omega_{S}\right)$, we can write $\omega_{s, T}=\phi_{s, T} \omega_{S}$ for some positive functions $\phi_{s, T}$ on $S$ such that $\phi_{0, T}=1$. Then, we have

$$
\left.\frac{d^{k}}{d s^{k}}\right|_{s=0} \omega_{s, T}=\phi_{0, T}^{(k)} \omega_{S}
$$

On the other hand, since $\left(h_{s, T}, \omega_{s, T}\right)$ are solutions to $\mathbf{L}_{\mathbf{s}, \mathbf{1}} \oplus \mathbf{L}_{\mathbf{s}, \mathbf{2}}=0$ $\bmod V_{1}$, there is a function $\mathbf{c}(s, T)$ taking values in $V_{1}$ with $\mathbf{c}(0, T)=0$ 
so that

$$
F_{s, T} \wedge \omega_{s, T}=h_{s, T}^{-1 / 2} \mathbf{c}(s, T) h_{s, T}^{1 / 2} .
$$

We can write

$$
\left.\mathbf{c}(s, T)=\operatorname{diag}\left(M_{1}(s, T), M_{2}(s, T),-\frac{\left(M_{1}(s, T)\right.}{2}+\frac{M_{2}(s, T)}{2}\right) I_{3}\right) \cdot \omega_{S}^{2},
$$

where $M_{i}(s, T)$ is the function only depending on $s$ and $T$.

At first, we compute $\mathbf{r}_{i}^{(k)}(0, T)$ for any $T$ and $k \leq m-1$. When $k \leq$ $m-1, A_{0}^{(k)}=0$. Then,

$$
F_{0, T}^{(k)}=\sum_{l=0}^{k} C_{k}^{l} D_{0}^{\prime \prime}\left[D_{0}^{\prime} h_{0, T}^{(l)} \cdot\left(h_{0, T}^{-1}\right)^{(k-l)}\right] .
$$

Because $D_{0}^{\prime} h_{T}=0, \dot{F}_{0, T}=D_{0}^{\prime \prime}\left[D_{0}^{\prime} \dot{h}_{0, T} \cdot h_{T}^{-1}\right]$ and

$$
\left.\frac{d}{d s}\right|_{s=0}\left(h_{s, T}^{-1 / 2} F_{s, T} h_{s, T}^{1 / 2}\right)=h_{0, T}^{-1 / 2} \dot{F}_{0, T} h_{0, T}^{1 / 2} .
$$

We also have $F_{0, T} \wedge \omega_{S}=0$. Combining these equalities with (8.10),

$$
\dot{\mathbf{r}}_{i}(0, T)=\int_{S} T_{i}^{-1 / 2}\left[\dot{F}_{0, T}\right]_{i} T_{i}^{1 / 2} \wedge \omega_{S}+\int_{S} T^{-1 / 2}\left[F_{0, T}\right]_{i} T^{1 / 2} \wedge \dot{\omega}_{S}=0 .
$$

On the other hand, taking derivative of $s$ at $s=0$ to (8.11) and couple $\mathbf{c}(0, T)=0$, we have

$$
\dot{F}_{0, T} \wedge \omega_{S}=h_{T}^{1 / 2} \dot{\mathbf{c}}(0, T) h_{T}^{-1 / 2}
$$

and then

$$
\left[\dot{F}_{0, T}\right]_{i} \wedge \omega_{S}=[\dot{\mathbf{c}}(0, T)]_{i}=\dot{M}_{i}(0, T) \omega_{S}^{2}
$$

From

$$
\int_{S} \dot{M}_{i}(0, T) \omega_{S}^{2}=\int_{S}\left[\dot{F}_{0, T}\right]_{i} \wedge \omega_{S}=0,
$$

we get $\dot{M}_{i}(0, T)=0$. So, we get

$$
\dot{\mathbf{c}}(0, T)=0 \quad \text { and } \quad \dot{F}_{0, T} \wedge \omega_{S}=\left(D_{0}^{\prime \prime} D_{0}^{\prime} \dot{h}_{0, T}\right) \cdot h_{0, T}^{-1} \wedge \omega_{S}=0 .
$$

Thus,

$$
\dot{h}_{0, T} \in V_{0} \quad \text { and } \quad D_{0}^{\prime} \dot{h}_{0, T}=0 .
$$


In this way, we can prove that

$$
\begin{aligned}
\mathbf{r}_{i}^{(k)}(0, T) & =0, \quad \mathbf{c}^{(k)}(0, T)=0, \quad F_{0, T}^{(k)} \wedge \omega_{S}=0, \\
D_{0}^{\prime} h_{0, T}^{(k)} & =0 \text { for any } k \leq m-1 .
\end{aligned}
$$

When $k=m$, because of (8.13) and (8.10), we obtain

$$
\left.\frac{d^{m}}{d s^{m}}\right|_{s=0}\left(h_{s, T}^{-1 / 2} F_{s, T} h_{s, T}^{1 / 2} \wedge \omega_{s, T}\right)=h_{0, T}^{-1 / 2} F_{0, T}^{(m)} h_{0, T}^{1 / 2} \wedge \omega_{S}
$$

and

$$
F_{0, T}^{(m)}=\left(D_{0}^{\prime \prime}+D_{0}^{\prime}\right)\left(A_{0}^{(m)}-A_{0}^{*(m)}\right)+D_{0}^{\prime \prime}\left[D_{0}^{\prime} h_{0, T}^{(m)}\right] \cdot h_{0, T}^{-1} .
$$

Then, we get

$$
\mathbf{r}_{i}^{(m)}(0, T)=\int_{S}\left[F_{0, T}^{(m)}\right]_{i} \wedge \omega_{S}=0
$$

Because $F_{0, T}^{(k)} \wedge \omega_{S}=0, \mathbf{c}^{(k)}(0, T)=0$ for $k \leq m-1$, from (8.11), we get

$$
F_{0, T}^{(m)} \wedge \omega_{S}=h_{T}^{1 / 2} \mathbf{c}^{(m)}(0, T) h_{T}^{-1 / 2}
$$

So, using the same method of case $k=1$, we still have

$$
\mathbf{c}^{(m)}(0, T)=F_{0, T}^{(m)} \wedge \omega_{S}=0 .
$$

When $k<m, A_{0}^{(k)}=0$ and we have proven $D_{0}^{\prime} h_{0, T}^{(k)}=0$. By direct computation, we see

$$
\begin{aligned}
F_{0, T}^{(m+k)}= & \left(D_{0}^{\prime \prime}+D_{0}^{\prime}\right)\left(A_{0}^{(m+k)}-A_{0}^{*(m+k)}\right) \\
& +D_{0}^{\prime \prime}\left(\left.\frac{d^{m+k}}{d s^{m+k}}\right|_{s=0}\left(D_{s}^{\prime} h_{s, T} \cdot h_{s, T}^{-1}\right)\right) .
\end{aligned}
$$

Then, we still can get

$$
\mathbf{r}_{i}^{(m+k)}(0, T)=0, \quad \mathbf{c}^{(m, k)}(0, T)=0, \quad F_{0, T}^{(m+k)} \wedge \omega_{S}=0 \text { for } k<m .
$$

At last we compute $\mathbf{r}^{(2 m)}(0, T)$. Directly computing, we get

$$
\begin{aligned}
F_{0, T}^{(2 m)}= & \left(D_{0}^{\prime \prime}+D_{0}^{\prime}\right)\left(A_{0}^{(2 m)}-A_{0}^{*(2 m)}\right)-C_{2 m}^{m}\left(A_{0}^{(m)}-A_{0}^{*(m)}\right) \wedge\left(A_{0}^{(m)}-A_{0}^{*(m)}\right) \\
& -C_{2 m}^{m}\left[A_{0}^{(m)}, d^{m} /\left.d s^{m}\right|_{s=0}\left(D_{s}^{\prime} h_{s, T} \cdot h_{s, T}^{-1}\right)\right] \\
& +D_{0}^{\prime \prime}\left(d^{2 m} /\left.d s^{2 m}\right|_{s=0}\left(D_{s}^{\prime} h_{s, T} \cdot h_{s, T}^{-1}\right)\right.
\end{aligned}
$$


and

$$
\begin{aligned}
& {\left[A_{0}^{(m)},\left.\frac{d^{m}}{d s^{m}}\right|_{s=0}\left(D_{s}^{\prime} h_{s, T} \cdot h_{s, T}^{-1}\right)\right]} \\
& \quad=\left[A_{0}^{(m)},\left[A_{0}^{*(m)}, h_{0, T}\right] h_{0, T}^{-1}\right]+\left[A_{0}^{(m)}, D_{0}^{\prime} h_{0, T}^{(m)} \cdot h_{0, T}^{-1}\right] .
\end{aligned}
$$

Then from $\quad d^{2 m} /\left.d s^{2 m}\right|_{s=0}\left(h_{s, T}^{-1 / 2} F_{s, T} h_{s, T}^{1 / 2} \wedge \omega_{S}\right)=h_{0, T}^{-1 / 2} F_{0, T}^{(2 m)} h_{0, T}^{1 / 2} \wedge \omega_{S}$, we see

$$
\begin{aligned}
\mathbf{r}_{i}^{(2 m)}(0, T)= & -C_{2 m}^{m} \int_{S}\left[\left(A_{0}^{(m)}-A_{0}^{*(m)}\right) \wedge\left(A_{0}^{(m)}-A_{0}^{*(m)}\right)\right]_{i} \\
& -C_{2 m}^{m} \int_{S}\left[\left[A_{0}^{(m)},\left[A_{0}^{*(m)}, h_{0, T}\right] h_{0, T}^{-1}\right]\right]_{i} \\
& -C_{2 m}^{m} \int_{S}\left[\left[A_{0}^{(m)}, D_{0}^{\prime} h_{0, T}^{(m)} \cdot h_{0, T}^{-1}\right]\right]_{i} .
\end{aligned}
$$

The last term is zero because $D_{0}^{\prime \prime *} A_{0}^{(m)}=0$ and Lemma 2.3 of [5]. Using

$$
A_{0}^{(m)}=\left(\begin{array}{ccc}
0 & 0 & C_{13} \\
0 & 0 & C_{23} \\
C_{31} & C_{32} & C_{33}
\end{array}\right) \quad \text { and } \quad H_{0, T}=\left(\begin{array}{ccc}
T_{1} & 0 & 0 \\
0 & T_{2} & 0 \\
0 & 0 & T_{1}^{-1 / 2} T_{2}^{-1 / 2} I_{3}
\end{array}\right)
$$

one computes

$$
\begin{aligned}
& {\left[\left[A_{0}^{(m)},\left[A_{0}^{*(m)}, h_{0, T}\right] h_{0, T}^{-1}\right]\right]_{1}} \\
& \quad=\left(1-T_{1}^{-3 / 2} T_{2}^{-1 / 2}\right) C_{13} \wedge C_{13}^{*}+\left(1-T_{1}^{3 / 2} T_{2}^{1 / 2}\right) C_{31}^{*} \wedge C_{31}
\end{aligned}
$$

and

$$
-\left[\left(A_{0}^{(m)}-A_{0}^{*(m)}\right) \wedge\left(A_{0}^{(m)}-A_{0}^{*(m)}\right)\right]_{1}=C_{13} \wedge C_{13}^{*}+C_{31}^{*} \wedge C_{31} .
$$

Therefore,

$$
\mathbf{r}_{1}^{(2 m)}(0, T)=-C_{2 m}^{m} \int_{S}\left(T_{1}^{-3 / 2} T_{2}^{-1 / 2} C_{13} \wedge C_{13}^{*}+T_{1}^{3 / 2} T_{2}^{1 / 2} C_{31}^{*} \wedge C_{31}\right) \wedge \omega_{S} .
$$

With the same reason, we have

$$
\mathbf{r}_{2}^{(2 m)}(0, T)=-C_{2 m}^{m} \int_{S}\left(T_{1}^{-1 / 2} T_{2}^{-3 / 2} C_{23} \wedge C_{23}^{*}+T_{1}^{1 / 2} T_{2}^{3 / 2} C_{32}^{*} \wedge C_{32}\right) \wedge \omega_{S}
$$


Let $B_{i 3}=\sqrt{-1} \int_{S} C_{i 3} \wedge C_{i 3}^{*} \wedge \omega_{S}$ and $B_{3 i}=-\sqrt{-1} \int_{S} C_{3 i}^{*} \wedge C_{3 i} \wedge \omega_{S}$ for $i=$ 1,2. By our assumption, we have $B_{i 3}>0$ and $B_{3 i}>0$. Then,

$$
\begin{aligned}
& \mathbf{r}_{1}^{(2 m)}(0, T)=\sqrt{-1} C_{2 m}^{m}\left\{B_{13} T_{1}^{-3 / 2} T_{2}^{-1 / 2}-B_{31} T_{1}^{3 / 2} T_{2}^{1 / 2}\right\} \\
& \mathbf{r}_{2}^{(2 m)}(0, T)=\sqrt{-1} C_{2 m}^{m}\left\{B_{23} T_{1}^{-1 / 2} T_{2}^{-3 / 2}-B_{32} T_{1}^{1 / 2} T_{2}^{3 / 2}\right\} .
\end{aligned}
$$

Clearly, $\mathbf{r}_{i}^{(2 m)}\left(0, T_{0}\right)=0$ if

$$
T_{0}=\left(T_{01}, T_{02}\right)=\left(\left(\frac{B_{13}}{B_{31}}\right)^{3 / 8}\left(\frac{B_{23}}{B_{32}}\right)^{-1 / 8},\left(\frac{B_{13}}{B_{31}}\right)^{-1 / 8}\left(\frac{B_{23}}{B_{32}}\right)^{3 / 8}\right) .
$$

Then, we define the map $G: B_{\epsilon}\left(T_{0}\right) \rightarrow S^{1}(1)$ by

$$
G(T)=\frac{\left(\mathbf{r}_{1}^{2 m}(0, T), \mathbf{r}_{2}^{2 m}(0, T)\right)}{\left\|\left(\mathbf{r}_{1}^{2 m}(0, T), \mathbf{r}_{2}^{2 m}(0, T)\right)\right\|}
$$

or

$$
G\left(\left(T_{1}, T_{2}\right)\right)=\frac{\left(B_{13} T_{1}^{-1}-B_{31} T_{1}^{2} T_{2}^{1}, B_{23} T_{2}^{-1}-B_{32} T_{1}^{1} T_{2}^{2}\right)}{\left\|\left(B_{13} T_{1}^{-1}-B_{31} T_{1}^{2} T_{2}^{1}, B_{23} T_{2}^{-1}-B_{32} T_{1}^{1} T_{2}^{2}\right)\right\|}
$$

Then it is easily proven that for $\epsilon$ small enough (here $\epsilon<1$ ), $G$ is homotopic to

$$
G_{1}(T)=\frac{\left(B_{13} T_{1}^{-1}, B_{23} T_{2}^{-1}\right)}{\left\|\left(B_{13} T_{1}^{-1}, B_{23} T_{2}^{-1}\right)\right\|}=\frac{\left(B_{13} T_{2}, B_{23} T_{1}\right)}{\left\|\left(B_{13} T_{2}, B_{23} T_{1}\right)\right\|} .
$$

Thus, $\operatorname{deg} G=\operatorname{deg} G_{1}=-1$. Then, as a discussion of the proof of Theorem 4.3 in paper [5], we see that the map for $\epsilon$ small enough,

$$
\left(\mathbf{r}_{1}, \mathbf{r}_{2}\right)(s, .): B_{\epsilon}\left(T_{0}\right) \rightarrow \mathbb{R}^{2}, \quad s \in\left(0, a^{\prime}\right)
$$

attains value $0 \in \mathbb{R}^{2}$ for all $s \in\left(0, a^{\prime}\right)$ in $B_{\epsilon}\left(T_{0}\right)$. So for sufficiently small $s$, there are solutions $\left(h_{s}, e^{\phi} \omega_{S}\right)$ to $\mathbf{L}_{\mathbf{s}}=0$ near $\left(h_{T_{0}}, \omega_{S}\right)$. From our definition of $\mathbf{L}_{\mathbf{s}, \mathbf{1}}$, we know that $h_{s, T}$ is the Hermitian-Yang-Mills solution on $\left(E, D_{s}\right)$. From Proposition 8.1 for $\mathbf{L}_{\mathbf{s}}$, we have obtained an irreducible solution of Strominger's system on non-Kähler manifold $X$. Hence, we have proven the following:

Theorem 8.4. Let $\left(E, H_{0}, S, \omega_{S}\right)$ be as before. Fix the holomorphic structure $D_{0}^{\prime \prime}$ of $E$. Then there is a smooth deformation $D_{s}^{\prime \prime}$ of $\left(E, D_{0}^{\prime \prime}\right)$ so that 
there are Hermitian-Yang-Mills metrics $H_{s}$ on $\left(E, D_{s}\right)$ and smooth function $\phi_{s}$ on $S$ such that

$$
\left(V=\pi^{*} E, \pi^{*} D_{s}^{\prime \prime}, \pi^{*} H_{s}, \tilde{\omega}_{s}=\pi^{*}\left(e^{u+\phi_{s}} \omega_{S}\right)+\frac{\sqrt{-1}}{2} \theta \wedge \bar{\theta}\right)
$$

are the irreducible solutions to Strominger's system on $X$. Moreover, $\lim _{s \rightarrow 0}$ $\phi_{s}=0$ and $\lim _{s \rightarrow 0} H_{s}$ is a regular reducible Hermitian-Yang-Mills connection on $E=L_{1} \oplus L_{2} \oplus T S$.

\section{Acknowledgments}

J. X. Fu would like to thank Professor J. Li for interesting discussions and helps. Proposition 8.3 is due to J. Li. J. X. Fu is supported in part by NSFC grant 10471026. S. T. Yau is supported in part by NSF grants DMS-0306600, DMS-0354737 and DMS-0244462.

\section{References}

[1] P. Candelas, G. Horowitz, A. Strominger, and E. Witten, Vacuum configurations for superstrings, Nucl. Phys. B 258 (1985), no. 1, 46-74.

[2] J. X. Fu and S.-T. Yau, The theory of superstring with flux on nonKähler manifolds and the complex Monge-Ampère equation. Preprint.

[3] E. Goldstein and S. Prokushkin, Geometric model for complex nonKähler manifolds with SU(3) structure, Comm. Math. Phys. 251 (2004), no. 1, 65-78.

[4] P. Hořava and E. Witten, Eleven-dimensional supergravity on a manifold with boundary. Nucl. Phys. B 475 (1996), 94-114.

[5] J. Li and S.-T. Yau, The existence of supersymmetric string theory with torsion. J. Diff. Geom. 70 (2005), no.1, 143-181.

[6] M. L. Michelsohn, On the existence of special metrics in complex geometry. Acta Math. 149 (1982), 261-295.

[7] A. Strominger, Superstings with torsion. Nucl. Phys. B 274 (1986), no. 2, 253-284.

[8] A. Strominger, Heterotic solution. Nucl. Phys. B 343 (1990), no. 1, $167-184$. 
[9] K. Uhlenbeck and S.-T. Yau, On the existence of Hermitian-YangMills connections in stable vector bundles. Comm. Pure Appl. Math. 39 (1986), no. S, suppl., S257-S293.

[10] R. O. Wells, Jr, Differential analysis on complex manifolds, World Publishing Corporation, Springer-Verlag, New York, Berlin, Heidelberg, Tokyo, 1979.

[11] E. Witten, New issues in manifolds of SU(3) holonomy. Nucl. Phys. B 268 (1986), no. 1, 79-112.

[12] S.-T. Yau, On the Ricci curvature of a compact Kähler manifold and the complex Monge-Ampère equation. I, Comm. Pure Appl. Math. 31 (1978), 339-411.

School of Mathematical Sciences

FUDAN UNIVERSITY

Shanghai 200433

People's Republic of China

E-mail address: majxfu@fudan.edu.cn

Department of Mathematics

HARVARD UNIVERSITY

CAmbridge, MA 02138

E-mail address: yau@math.harvard.edu

ReCeived April 10, 2006 
